CERN-PPE/97-059

26 May 1997

\title{
THE NOMAD EXPERIMENT AT THE CERN SPS
}

\author{
The NOMAD Collaboration
}

J. Altegoer ${ }^{6}$ M. Anfreville ${ }^{20}$ C. Angelini ${ }^{18}$ P. Astier ${ }^{15}$ M. Authier ${ }^{20}$ D. Autiero ${ }^{9}$ A. Baldisseri ${ }^{20}$ M. Baldo-Ceolin ${ }^{14}$ G. Ballocchi ${ }^{9}$ M. Banner ${ }^{15}$ S. Basa $^{10} \quad$ G. Bassompierre ${ }^{2}$ K. Benslama ${ }^{10}$ I. Bird ${ }^{10}$ B. Blumenfeld ${ }^{3}$ F. Bobisut ${ }^{14}$ J. Bouchez ${ }^{20}$ S. Boyd ${ }^{22}$ A. Bueno ${ }^{1,4}$ S. Bunyatov ${ }^{7}$ L. Camilleri ${ }^{9}$ A. Cardini ${ }^{11}$ A. Castera ${ }^{15}$ P.W. Cattaneo ${ }^{16}$ V. Cavasinni ${ }^{18}$ V. Cavestro $\dagger^{14}$ O. Cloué ${ }^{20}$ G. Conforto ${ }^{23}$ C. Conta ${ }^{16}$ R. Cousins ${ }^{11}$ A. De Santo ${ }^{18}$ T. Del Prete ${ }^{18}$ L. Di Lella ${ }^{9}$ T. Dignan ${ }^{4}$ E. do Couto e Silva ${ }^{9}$ I.J. Donnelly ${ }^{21,22}$ J. Dumarchez ${ }^{15}$ L. Dumps ${ }^{9} \quad$ C. Engster ${ }^{9}$

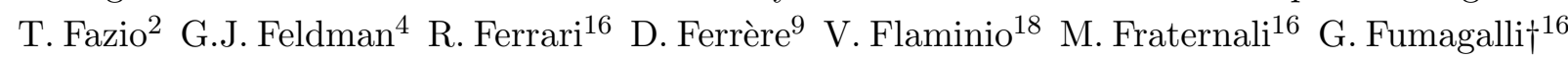
J.-M. Gaillard ${ }^{2}$ P. Galumian ${ }^{10}$ E. Gangler ${ }^{15}$ A. Geiser ${ }^{9}$ D. Geppert ${ }^{6}$ D. Gibin ${ }^{14}$ S. Gninenko ${ }^{13}$ J.-J. Gomez-Cadenas ${ }^{1,9}$ J. Gosset ${ }^{20}$ C. Gößling ${ }^{6}$ M. Gouanère ${ }^{2} \quad$ A. Grant ${ }^{9} \quad$ G. Graziani ${ }^{8}$

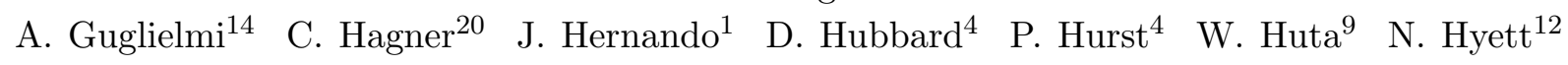
E. Iacopini ${ }^{8}$ C. Joseph ${ }^{10}$ D. Kekez ${ }^{24}$ M. Kirsanov ${ }^{8,13}$ B. Khomenko ${ }^{9}$ O. Klimov ${ }^{7}$ A. Kovzelev ${ }^{13}$ V. Kuznetsov ${ }^{7}$ A. Lanza ${ }^{16}$ L. La Rotonda ${ }^{5}$ M. Laveder ${ }^{14}$ C. Lazzeroni ${ }^{18}$ A. Letessier-Selvon ${ }^{15}$ J.-M. Levy ${ }^{15}$ L. Linssen ${ }^{9}$ A. Ljubičić ${ }^{24}$ J. Long $^{3}$ A. Lupi ${ }^{8}$ E. Manola-Poggioli ${ }^{2}$ A. Marchionni ${ }^{8}$ F. Martelli ${ }^{23}$ J.-P. Mendiburu ${ }^{2}$ J.-P. Meyer ${ }^{20}$ M. Mezzetto ${ }^{14}$ S.R. Mishra ${ }^{4}$ G.F. Moorhead ${ }^{12}$

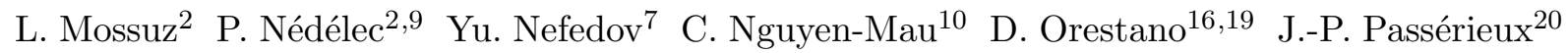
F. Pastore ${ }^{16,19}$ L.S. Peak ${ }^{22}$ E. Pennacchio ${ }^{23}$ J.-P. Perroud ${ }^{10}$ H. Pessard ${ }^{2}$ P. Petitpas ${ }^{2}$ R. Petti ${ }^{16}$ A. Placci ${ }^{9}$ H. Plothow-Besch ${ }^{9}$ A. Pluquet ${ }^{20} \quad$ J. Poinsignon ${ }^{20}$ G. Polesello ${ }^{16}$ D. Pollmann ${ }^{6}$ B.G. Pope ${ }^{9}$ B. Popov ${ }^{7,15}$ C. Poulsen ${ }^{12}$ P. Rathouit ${ }^{20}$ G. Renzoni ${ }^{18}$ C. Roda ${ }^{9}$ A. Rubbia ${ }^{9}$ F. Salvatore ${ }^{16}$ K. Schahmaneche ${ }^{15}$ B. Schmidt ${ }^{6}$ A. Sconza ${ }^{14}$ M. Serrano ${ }^{15}$ M.E. Sevior ${ }^{12}$ D. Sillou ${ }^{2}$ C. Sobczynski ${ }^{9}$ F.J.P. Soler ${ }^{22}$ G. Sozzi ${ }^{10}$ D. Steele ${ }^{3}$ M. Steininger ${ }^{10}$ M. Stipčević ${ }^{24}$ T. Stolarczyk ${ }^{20}$ G.N. Taylor ${ }^{12}$ V. Tereshchenko ${ }^{7}$ A. Toropin ${ }^{13}$ A.-M. Touchard ${ }^{15}$ S.N. Tovey ${ }^{12}$ M.-T. Tran ${ }^{10}$ E. Tsesmelis ${ }^{9}$ J. Ulrichs ${ }^{22}$ V. Uros $^{15}$ M. Valdata-Nappi ${ }^{5,17}$ V. Valuev ${ }^{7,2}$ F. Vannucci ${ }^{15}$ K.E. Varvell ${ }^{21,22}$ M. Veltri ${ }^{23}$ V. Vercesi ${ }^{16}$ D. Verkindt ${ }^{2}$

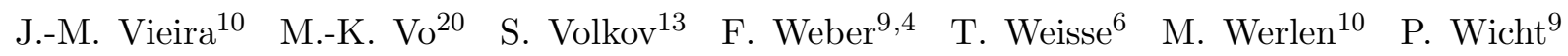
F.F. Wilson ${ }^{9}$ L.J. Winton ${ }^{12}$ B.D. Yabsley ${ }^{22}$ and H. Zaccone ${ }^{20}$ 


\footnotetext{
1) Univ. of Massachusetts, Amherst, MA, USA

2) LAPP, Annecy, France

3) Johns Hopkins Univ., Baltimore, MD, USA

4) Harvard Univ., Cambridge, MA, USA

5) Univ. of Calabria and INFN, Cosenza, Italy

6) Dortmund Univ., Dortmund, Germany

7) JINR, Dubna, Russia

8) Univ. of Florence and INFN, Florence, Italy

9) CERN, Geneva, Switzerland

10) University of Lausanne, Lausanne, Switzerland

11) UCLA, Los Angeles, CA, USA

12) University of Melbourne, Melbourne, Australia

13) Inst. Nucl. Research, INR Moscow, Russia

14) Univ. of Padova and INFN, Padova, Italy

15) LPNHE, Univ. of Paris, Paris VI and VII, France

16) Univ. of Pavia and INFN, Pavia, Italy

17) Now at Perugia Univ., Perugia, Italy

18) Univ. of Pisa and INFN, Pisa, Italy

19) Now at Roma-III Univ., Rome, Italy

20) DAPNIA, CEA Saclay, France

21) ANSTO Sydney, Menai, Australia

22) University of Sydney, Sydney, Australia

23) Univ. of Urbino, Urbino, and INFN Florence, Italy

24) Rudjer Bošković Institute, Zagreb, Croatia

$\dagger$ Deceased
} 


\begin{abstract}
The NOMAD experiment is a short base-line search for $\nu_{\mu} \rightarrow \nu_{\tau}$ oscillations in the CERN neutrino beam. The $\nu_{\tau}$ 's are searched for through their charged-current interactions followed by the observation of the resulting $\tau^{-}$through its electronic, muonic or hadronic decays. These decays are recognized using kinematical criteria necessitating the use of a light target which enables the reconstruction of individual particles produced in the neutrino interactions. This paper describes the various components of the NOMAD detector: the target and muon drift chambers, the electromagnetic and hadronic calorimeters, the preshower and transition radiation detectors, and the veto and trigger scintillation counters. The beam and data acquisition system are also described. The quality of the reconstruction of individual particles is demonstrated through the ability of NOMAD to observe $\mathrm{K}_{\mathrm{s}}^{0}$ 's, $\Lambda^{0}$ 's and $\pi^{0}$ 's. Finally, the observation of $\tau^{-}$through its electronic decay being one of the most promising channels in the search, the identification of electrons in NOMAD is discussed.
\end{abstract}



The main goal of the NOMAD (Neutrino Oscillation MAgnetic Detector) experiment is to search for the appearance of tau neutrinos $\left(\nu_{\tau}\right)$ in the CERN SPS wideband neutrino beam. This beam has a mean energy of $24 \mathrm{GeV}$ and the predominant neutrino type is $\nu_{\mu}$. As a by-product the experiment can set limits on $\nu_{\mu} \rightarrow \nu_{\mathrm{e}}$ oscillations.

The NOMAD detector measures and identifies most of the particles, charged and neutral, produced in neutrino interactions within the detector. The active target is a set of drift chambers with a fiducial mass of about 2.7 tons and a low average density $\left(98.6 \mathrm{~kg} / \mathrm{m}^{3}\right)$. The detector is located in a dipole magnetic field of $0.4 \mathrm{~T}$ which allows the determination of the momenta of charged tracks via their curvature, with minimal degradation due to multiple scattering. The active target is followed by a Transition Radiation Detector (TRD) to identify electrons, an electromagnetic calorimeter including a preshower detector, a hadronic calorimeter, and muon chambers. This paper describes the detector in detail.

In three years' running at the CERN SPS, NOMAD should collect data with more than 1 million charged current (CC) $\nu_{\mu}$ events.

The $\nu_{\tau}$ will be searched for via its CC interactions: $\nu_{\tau}+\mathrm{N} \rightarrow \tau^{-}+\mathrm{X}$. Given the lifetime of the $\tau^{-}$and the energies considered here, the $\tau^{-}$will travel about $1 \mathrm{~mm}$ before decaying. The spatial resolution of NOMAD, while good, is not sufficient to recognize the non-zero impact parameter associated with such tracks. Instead, the decays of the $\tau^{-}$ will be identified using kinematic criteria, based on a precise measurement of the missing transverse momentum in the final state.

In order to be sensitive to a large fraction of the $\tau^{-}$decay modes and to be able to select events with high acceptance and low background, the NOMAD detector must be able to:

- Measure the momenta of charged particles in the drift chamber target with good precision.

- Identify and measure electrons and photons.

- Identify and measure muons.

- Achieve a high level of rejection against tracks which fake electrons and muons.

As will be discussed below the NOMAD detector is well on the way to achieving these goals.

In addition to searching for neutrino oscillations, the large sample of data in a detector with a target density of a hydrogen bubble chamber will permit NOMAD to explore many other processes involving neutrinos.

The next section of this paper describes the NOMAD subdetectors. The following section documents its excellent performance.

\section{THE DETECTOR AND BEAM}

The NOMAD detector [1] is shown schematically in Fig. 1 (side view) and Fig. 2 (top view). It consists of a number of subdetectors most of which are located in a dipole magnet [2] with a field volume of $7.5 \times 3.5 \times 3.5 \mathrm{~m}^{3}$. Moving downstream along the beam direction we find a veto counter, a front calorimeter, a large active target consisting of drift chambers, a transition radiation detector, a preshower, an electromagnetic calorimeter, a hadron calorimeter, and an iron filter followed by a set of large drift chambers used for muon identification. Upstream and downstream of the transition radiation detector 
two large scintillator hodoscopes provide a fast trigger. The magnetic field is horizontal, perpendicular to the neutrino beam direction, and has the value of $0.4 \mathrm{~T}$. The key features of each subdetector are given below. In many cases more detailed technical descriptions have been published or are in preparation.

Referring to Fig. 1, the coordinate system adopted for NOMAD has the $x$-axis into the plane of that figure, the $y$-axis directed up towards the top of the detector and the $z$-axis horizontal, approximately along the direction of the neutrino beam (the beam line points upwards, at an angle of $2.4^{\circ}$ to the $z$-axis).

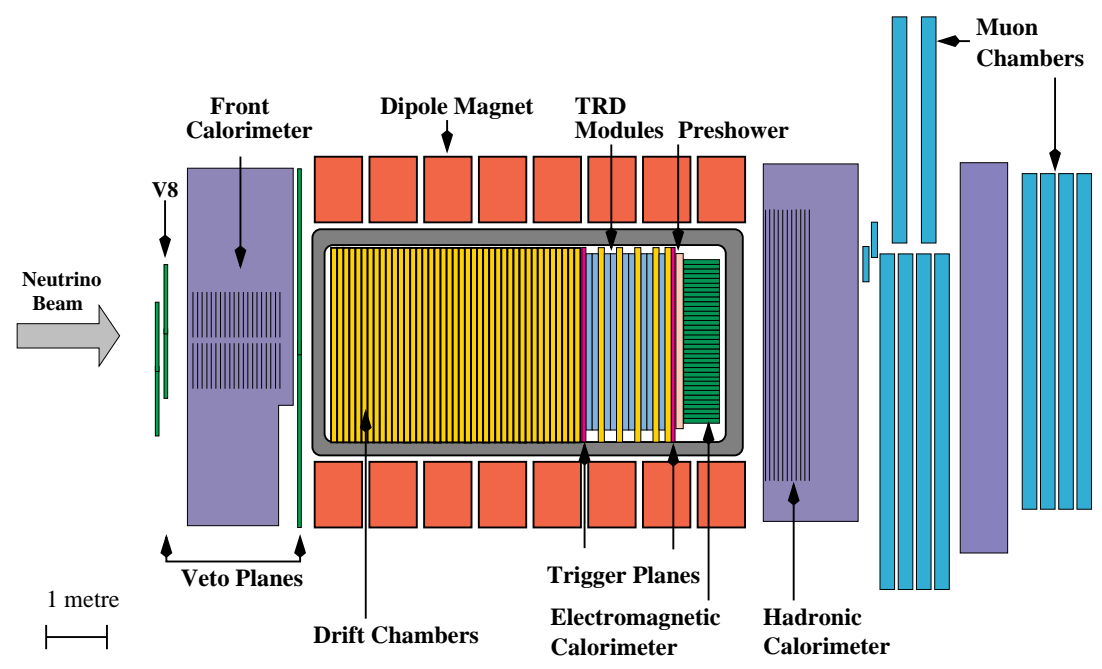

Figure 1: A side view of the NOMAD detector

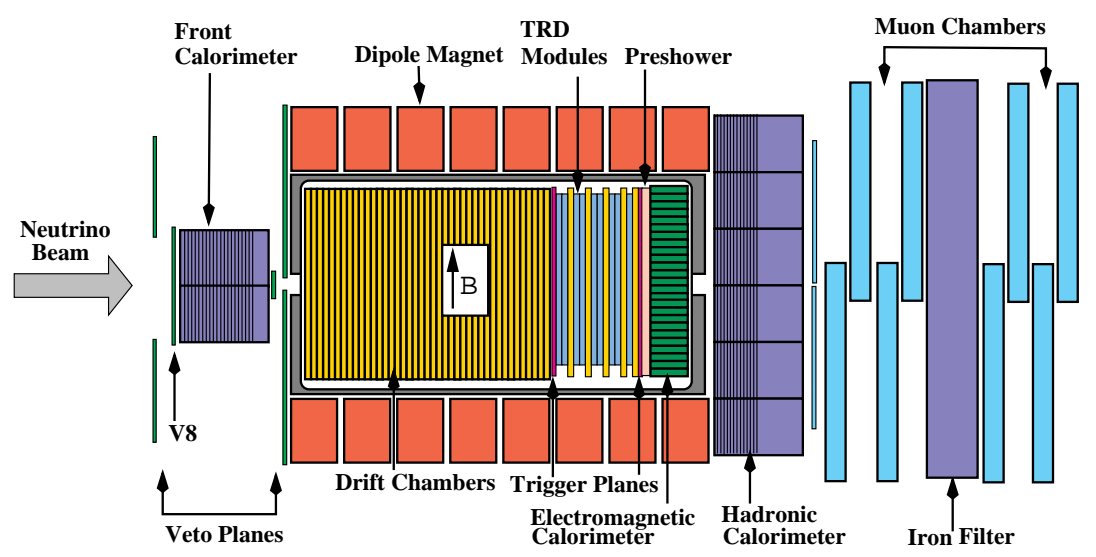

Figure 2: A top view of the NOMAD detector

\subsection{The neutrino beam}

The NOMAD detector is located at the CERN West Area Neutrino Facility (WANF) and is exposed to the SPS wideband neutrino beam which consists predominantly of $\nu_{\mu}$ 's. The beam line has been operating for nearly 20 years and was re-optimized in 1992 and 1993 [3] for the NOMAD and CHORUS experiments. Figure 3 shows a schematic layout of the WANF beam line. 

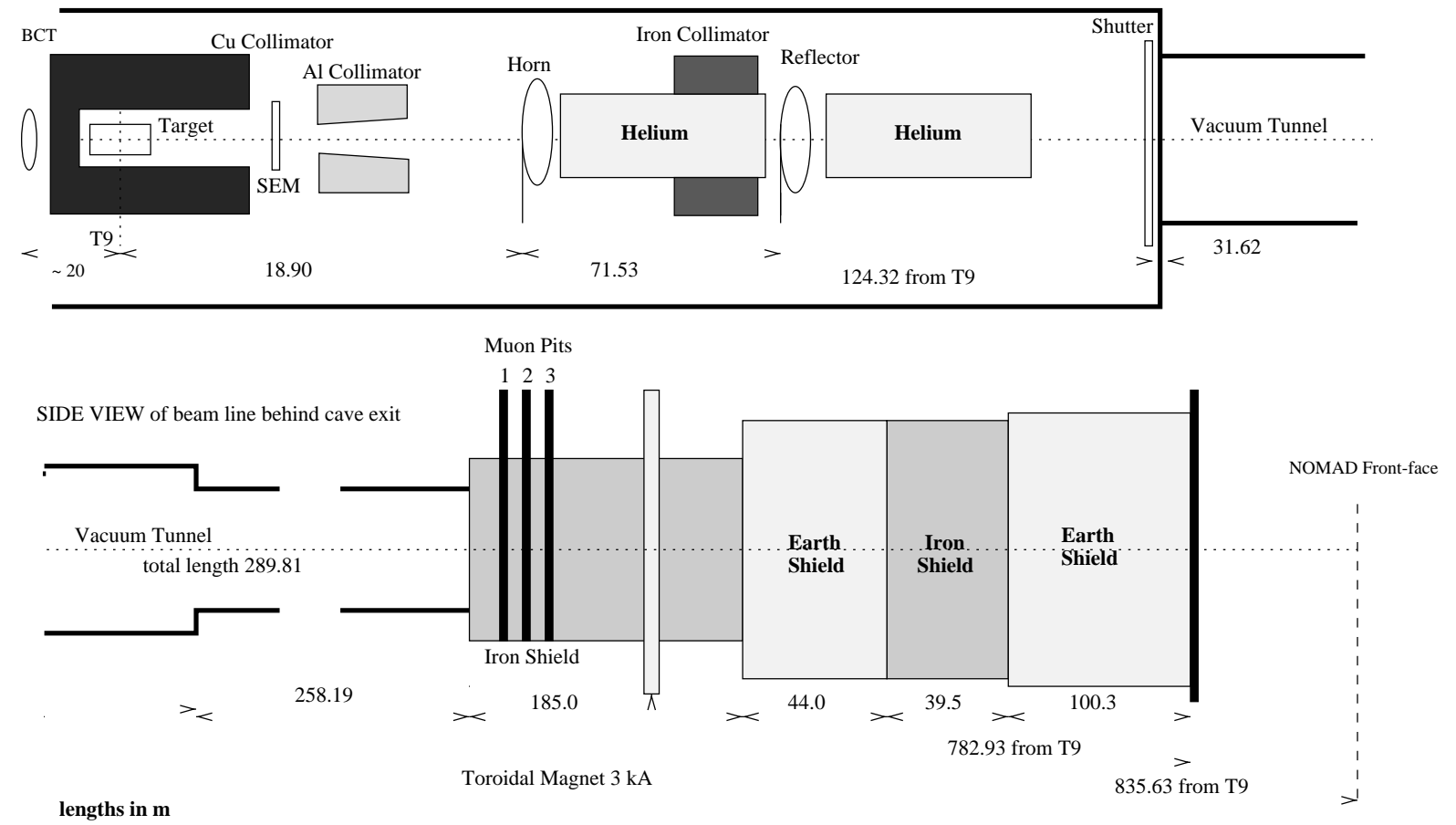

Figure 3: Schematic layout of the WANF beam line pointing out its main elements. Not drawn to scale. See the text for further explanation.

The Beam Current Transformer (BCT) upstream of the target measures the flux of incident protons, while the downstream Secondary Emission Chamber (SEM) measures the total flux of secondary particles in the forward direction. The shutter upstream of the vacuum decay tunnel is not present during normal beam operations, but is used to protect maintenance personnel working in that area should the thin window at the entrance of the vacuum decay tunnel rupture.

The neutrinos are primarily produced from the decays in flight of the secondary $\pi$ and $\mathrm{K}$ mesons originating from $450 \mathrm{GeV}$ protons impinging on a beryllium target. The SPS cycle repeats every $14.4 \mathrm{~s}$. The protons are extracted from the SPS in two $4 \mathrm{~ms}$ long spills (fast/slow extraction) separated by $2.6 \mathrm{~s}$ with a $2.0 \mathrm{~s}$ 'flat top'. The proton beam has a Gaussian shape with $\sigma \approx 0.5 \mathrm{~mm}$ at the target. The new beam line has been operating now for over three years with record intensities up to $1.5 \times 10^{13}$ protons in each of the two spills [4].

For the current experiments, a new target station has been installed keeping the same configuration of 11 beryllium rods separated by $9 \mathrm{~cm}$ gaps. Each rod is $10 \mathrm{~cm}$ long and $3 \mathrm{~mm}$ in diameter and is positioned longitudinally along the proton line. A new small-angle collimator was installed immediately after the target rods in order to better match the secondary particle beam to the entrance apertures of the magnetic elements downstream. In addition, the data acquisition and control system of the neutrino flux monitoring system were replaced with a modern configuration [5]. The region around the focusing elements has also been optimized to increase the neutrino intensity. The secondary pions and kaons are focused by a pair of coaxial magnetic lenses - the horn and reflector [6]. In such a system charged particles are deflected by the toroidal field between two coaxial conductors carrying equal and opposite currents so that the focusing of particles of one sign implies defocusing particles of the opposite sign. In order to harden the neutrino spectrum, the horn and reflector were displaced by about $8 \mathrm{~m}$ downstream to 
new positions of $20 \mathrm{~m}$ and $90 \mathrm{~m}$ from the target respectively. The higher neutrino energy increases the sensitivity of the experiments to detect charged current $\nu_{\tau}$ interactions, which have an energy threshold of $3.5 \mathrm{GeV}$.

In addition, the sections between the horn and reflector and between the reflector and decay tunnel, which were formerly open to air, have been enclosed in helium tubes of $80 \mathrm{~cm}$ diameter and total length of about $60 \mathrm{~m}$ in order to reduce the absorption of the secondary particles. A large-angle collimator has been installed between the horn and reflector to reduce the $\bar{\nu}$ contamination by intercepting the defocused negative secondaries before they decay.

The mesons are allowed to decay in a $290 \mathrm{~m}$ long vacuum tunnel. Shielding made from iron and earth follows to range out the muons and absorb the hadrons. A toroidal magnet, operated at $3 \mathrm{kA}$, located at the entrance of the iron shielding, bends muons which would pass outside the shielding either further outwards or back into the iron plug which provides sufficient absorption. The NOMAD (CHORUS) detectors are located at about $835 \mathrm{~m}(823 \mathrm{~m})$ from the target. The average distance between the meson decay point and NOMAD is $620 \mathrm{~m}$.

A neutrino beam monitoring system (the muon 'pits') [7], based on the detection of muon yields at several depths in the iron shield, is built into the line. The silicon detectors in these muon pits also provide an absolute flux measurement. The absolute calibration of the silicon detectors is performed periodically using emulsion measurements in the pits. An independent measurement of the flux is given by the number of protons incident on the target, estimated from a pair of beam current transformers located upstream of the target (BCT1 and BCT2).

A detailed GEANT [8] simulation of the beam line was used to predict the neutrino energy and radial position distributions at the NOMAD detector. These distributions, calculated for a fiducial area of $2.6 \times 2.6 \mathrm{~m}^{2}$ and for $10^{9}$ protons on target (p.o.t.) are shown in Figs. 4 and 5.

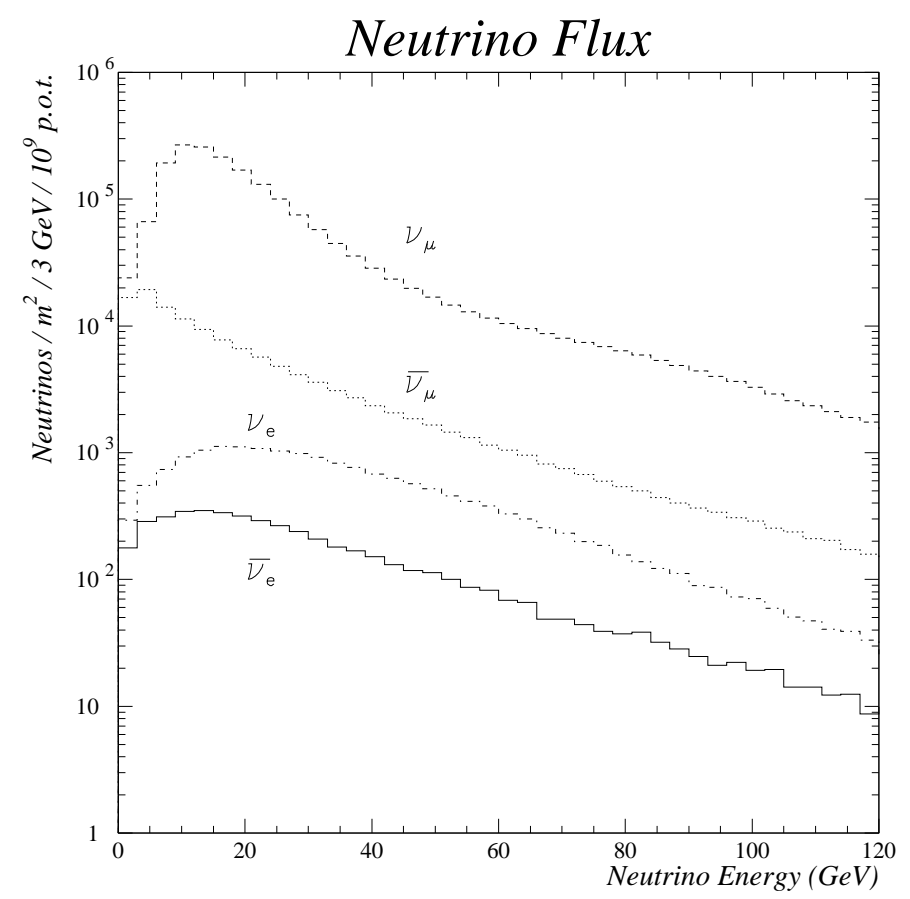

Figure 4: The predicted energy spectra of neutrinos at NOMAD 


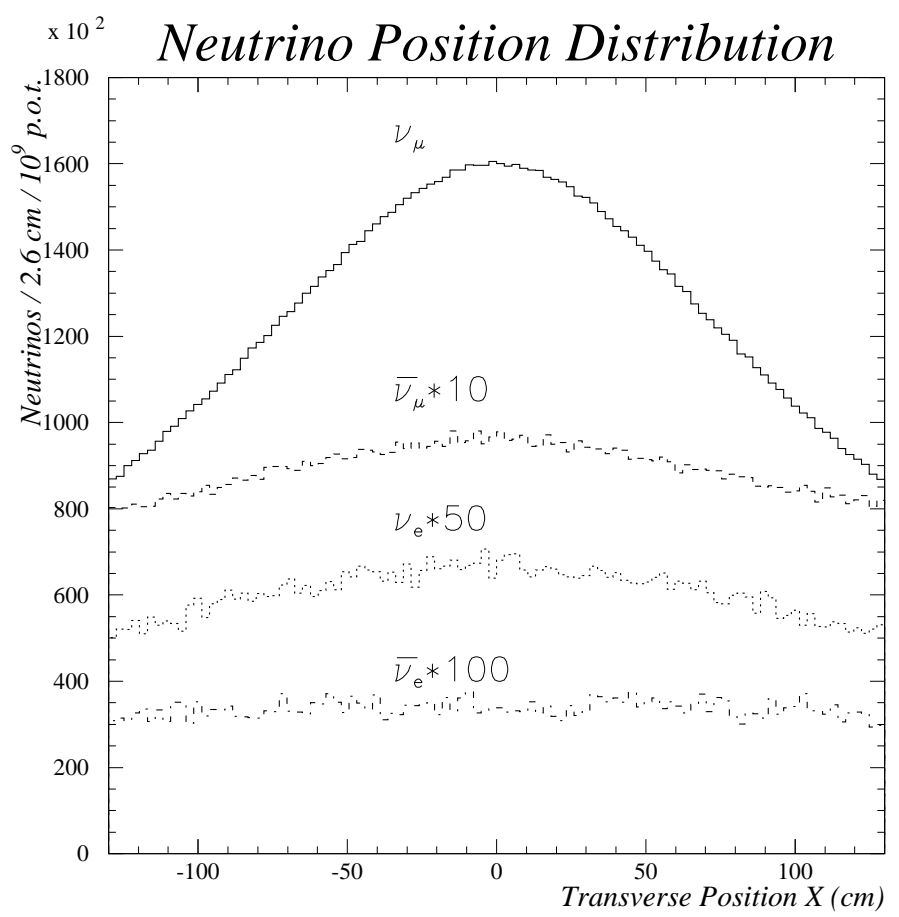

Figure 5: The predicted transverse position distribution of neutrinos at NOMAD in the horizontal direction. (Owing to the beam line symmetry similar spectra are obtained in the other transverse direction.)

The parameters of the various neutrino components are summarized in Table 1. The neutrino beam simulation is affected by uncertainties due mostly to the limited knowledge of the $\pi$ and $\mathrm{K}$ yields from the hadronic interactions in the beryllium target. The measurement by the SPY collaboration [9] of the production rates of $\pi$ and K mesons and of their ratio for $450 \mathrm{GeV}$ protons incident on a beryllium target will reduce these uncertainties.

The intrinsic $\nu_{\tau}$ component in the beam, from the prompt reaction:

$$
\mathrm{p}+\mathrm{N} \rightarrow \mathrm{D}_{\mathrm{s}}^{ \pm}+\mathrm{X}, \mathrm{D}_{\mathrm{s}}^{ \pm} \rightarrow \tau^{ \pm}+{ }^{(} \bar{\nu}_{\tau}^{)}
$$

followed by $\tau^{ \pm} \rightarrow \nu_{\tau}+\mathrm{X}$ has been calculated to be negligible [10, 11]. The relative number of $\nu_{\tau}$ 's produced from the above reaction and interacting via the $\mathrm{CC}$ in the fiducial volume of NOMAD has been calculated to be $(4-5) \times 10^{-6}$ the number of $\nu_{\mu} \mathrm{CC}$ events. After selection criteria, the resulting intrinsic $\nu_{\tau}$ signal is much less than one observed event in the total duration of the NOMAD experiment.

Table 1: Monte Carlo predictions at NOMAD for a fiducial area of $2.6 \times 2.6 \mathrm{~m}^{2}$ and $2.4 \times 10^{19}$ p.o.t.

\begin{tabular}{|c|c|c|c|}
\hline & Average $E_{\nu}(\mathrm{GeV})$ & Rel. flux abundance & $\nu$ CC events \\
\hline$\nu_{\mu}$ & 23.6 & 1.00 & $1.15 \times 10^{6}$ \\
\hline $\bar{\nu}_{\mu}$ & 22.7 & 0.07 & $0.39 \times 10^{5}$ \\
\hline$\nu_{\mathrm{e}}$ & 37.0 & $10^{-2}$ & $0.17 \times 10^{5}$ \\
\hline $\bar{\nu}_{\mathrm{e}}$ & 33.2 & $3 \times 10^{-3}$ & $0.22 \times 10^{4}$ \\
\hline
\end{tabular}




\subsection{Veto counters}

The veto system consists of an arrangement of 59 scintillation counters [12] covering an area of $5 \times 5 \mathrm{~m}^{2}$ at the upstream end of the NOMAD detector. The scintillators have a thickness of $2 \mathrm{~cm}$, a width of $21 \mathrm{~cm}$, and are of two lengths, $300 \mathrm{~cm}$ and $210 \mathrm{~cm}$. Most (56) of the counters are viewed at both ends by photomultipliers; the remaining (3) counters have single-ended readout. The counters are arranged in a geometry which provides optimal rejection of charged particles produced upstream of NOMAD, of those produced in neutrino interactions in the iron detector support, and of large-angle cosmic rays travelling in the same and in the opposite direction to the neutrino beam. A small fraction of interactions in the central region of the iron cannot be excluded, as the support beam for the steel structure (the 'basket') in which the central detector is mounted passes through the space in which scintillators would ideally be placed. Similarly, interactions in the upstream part of the magnet coil are not vetoed, but can be distinguished from target interactions by reconstruction of the event vertex.

The two photomultiplier outputs connected to each scintillation counter are fed via discriminators to the inputs of mean-timer modules [13], the timing of which provides an output signal independent in time of the position at which the detected charged particle traversed the counter. A single mean-timer module can take up to 16 inputs ( 8 pairs of discriminated photomultiplier outputs), and produces 8 outputs, which are passed to a 96-channel Fastbus TDC module. The unit used is a LeCroy 1876100 module with a bin width of 1 ns. In addition, each module produces a single output consisting of the logical OR of all eight output signals. For central detector triggers, the veto signal is formed by the combined OR of all mean-timer modules, excluding six counters at the upper-left and upper-right of the veto. In general, muons crossing these counters did not cross the NOMAD trigger planes, but their inclusion increased the dead time due to the veto. The signals from these counters are still recorded and are available off line. For triggers in the front calorimeter, which instruments the central region of the front detector support, the veto is formed from the subset of 10 counters (denoted $V_{8}$ ) mounted on the front face of the support, see Figs. 1 and 2.

The charged particle rejection efficiency of the NOMAD veto is constantly monitored and has remained stable at a level of 96-97\%. Averaged over the two neutrino spills, the contribution of the veto system to the overall dead time of NOMAD is $4 \%$.

\subsection{Front calorimeter}

The detector is suspended from iron pillars (the I's) at the two ends of the magnet. The front pillar was instrumented with scintillators to provide an additional massive active target for neutrino interactions. Physics topics to be addressed by this Front Calorimeter (FCAL) include multi-muon physics and searches for neutral heavy objects produced in neutrino interactions.

The FCAL consists of 23 iron plates which are $4.9 \mathrm{~cm}$ thick and separated by $1.8 \mathrm{~cm}$ gaps. Twenty out of the 22 gaps are instrumented with long scintillators [14] which are read out on both ends by 3 inch photomultipliers. The dimensions of the scintillators are $175 \times 18.5 \times 0.6 \mathrm{~cm}^{3}$. To achieve optimal light collection and a reasonable number of electronic channels, five consecutive scintillators along the beam axis are ganged together by means of twisted light guides and form a module. Ten such modules are placed above each other and form a stack. Along the beam axis are four such stacks, as shown in Fig. 6. The area of the FCAL 'seen' by the neutrino beam is $175 \times 190 \mathrm{~cm}^{2}$. The detector has a depth of about five nuclear interaction lengths and a total mass of about 17.7 tons. 


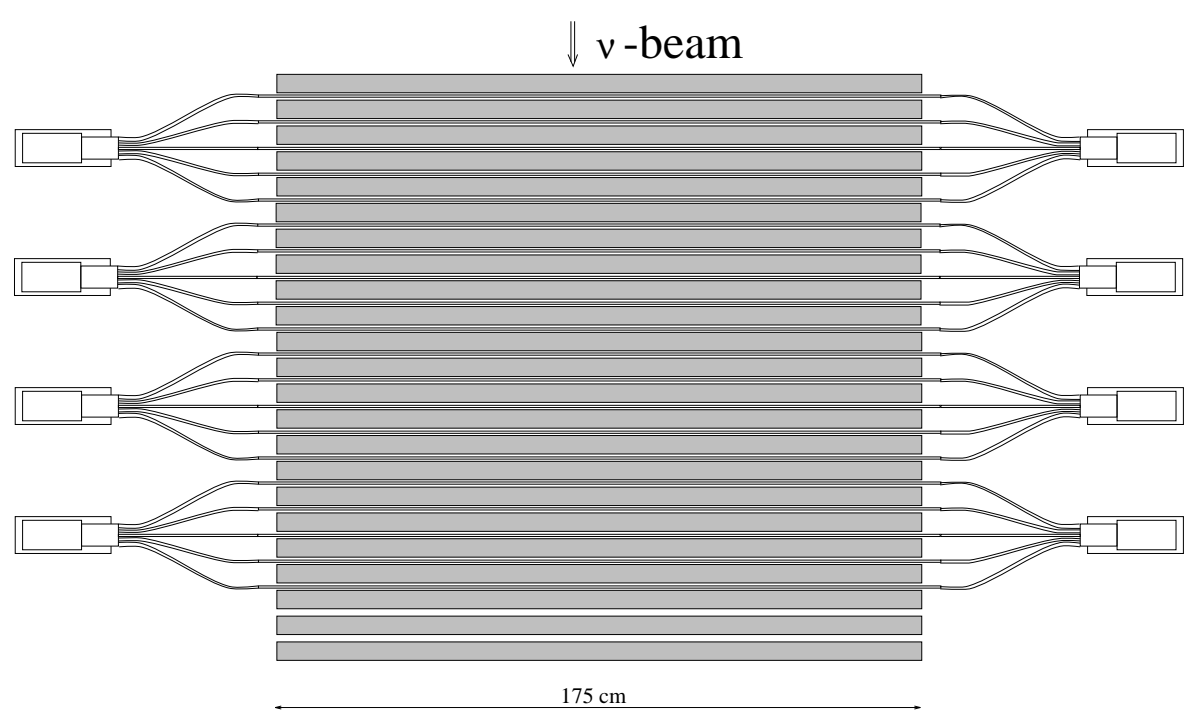

Figure 6: Top view of the FCAL

Since the FCAL is part of the structural components of the NOMAD detector, it cannot easily be calibrated in a test beam of known hadronic energy. The calibration procedure applied is therefore somewhat more sophisticated:

- A module-to-module calibration is achieved by determining the energy deposition by highly relativistic muons, a reasonable approximation of minimally ionizing particles (m.i.p.). These muons are copiously produced in the beam lines upstream of the NOMAD experiment and cross the detector in the time between the neutrino spills.

- The absolute energy scale (i.e. the hadronic energy equivalent of a m.i.p.) can be determined in several ways:

- A first-level calibration can be obtained from Monte Carlo simulation of neutrino interactions in the FCAL by comparing the energy deposit (calibrated in units of the m.i.p. energy deposit) with the simulated hadronic energy.

- A more accurate calibration can be performed by exploiting the fact that the shape of the $y_{\text {Bjorken-distribution in neutrino charged-current interactions is well }}$ known. Given the very precise momentum measurement of the muon produced in the interaction, a mismeasurement of the hadronic energy results in a distorted distribution in $y_{\text {Bjorken }}$. This method has been used successfully in previous neutrino experiments (see, for example Ref. [15]). The absolute energy scale in the FCAL has been determined by this method with a precision of $2 \%$. The results show that the Monte Carlo method mentioned above is accurate to $8 \%$.

A minimum-ionizing particle traversing the whole FCAL has an equivalent hadronic energy of $430 \mathrm{MeV}$.

\subsection{Drift chambers}

The drift chambers, which provide at the same time the target material and the tracking of particles, are a crucial part of the detector. They were designed with the conflicting requirements that their walls should be as heavy as possible in order to maximize the number of neutrino interactions and as light as possible in order to minimize multiple scattering of particles, secondary particle interactions, and photon conversions. To 
minimize the total number of radiation lengths for a given target mass, the chambers are made of low density and low atomic number materials; there is less than $1 \%$ of a radiation length between two consecutive measurements.

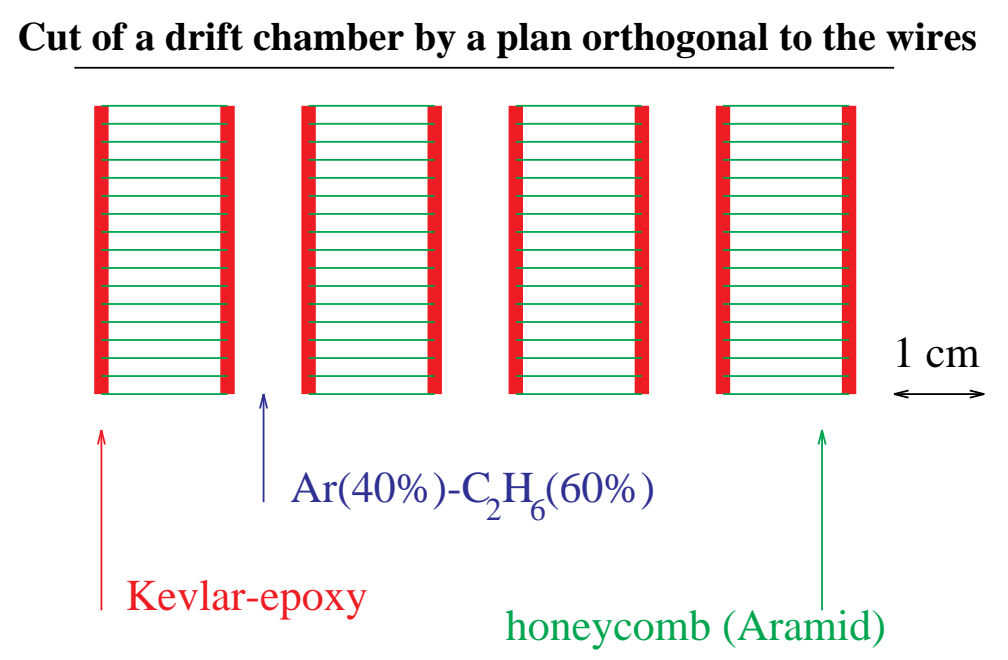

\section{Details of a drift cell}

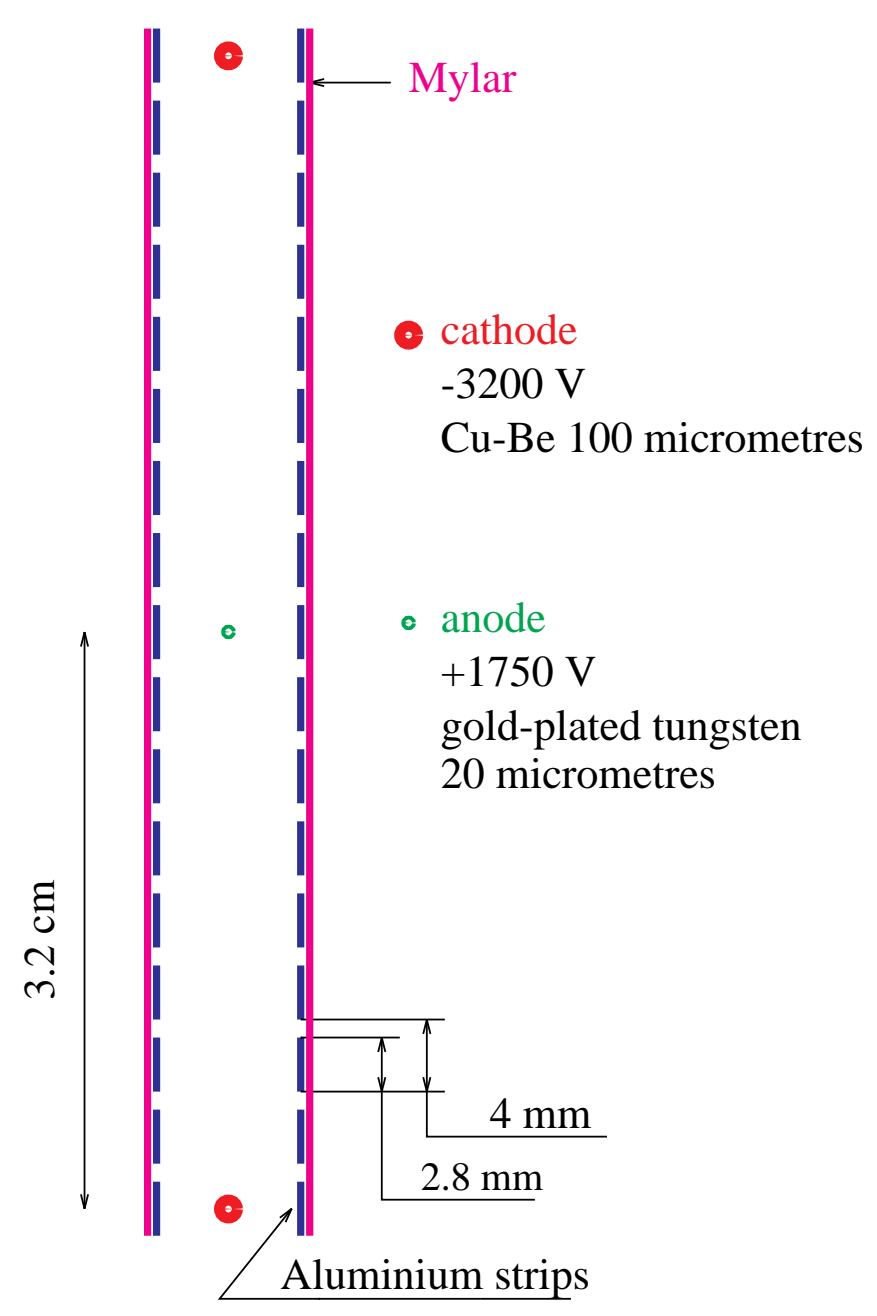

Figure 7: An overview of the drift chamber layout 
The chambers (see Fig. 7) are built on panels made of Aramid fibres in a honeycomb structure. These panels are sandwiched between two Kevlar-epoxy resin skins. These skins give the mechanical rigidity and flatness necessary over the large $\left(3 \times 3 \mathrm{~m}^{2}\right)$ surface area. Each drift chamber consists of four panels. The three $8 \mathrm{~mm}$ gaps between the panels are filled with an argon (40\%) - ethane (60\%) mixture at atmospheric pressure. Because the panels are not completely gas tight, the gas circulates permanently in a closed circuit with a purifier section that removes oxygen and water vapour.

The central gap is equipped with sense wires at 0 degrees and the outer gaps with wires at +5 and -5 degrees with respect to the magnetic field direction. These sense wires are $20 \mu \mathrm{m}$ in diameter and are made of gold-plated tungsten. They are interleaved with $100 \mu \mathrm{m}$ potential wires, made of $\mathrm{Cu}$-Be. These wires are equally spaced vertically to provide drift cells of $\pm 3.2 \mathrm{~cm}$ around each sense wire. Field-shaping aluminium strips, $2.8 \mathrm{~mm}$ wide, $12 \mu \mathrm{m}$ thick, and separated by $1.2 \mathrm{~mm}$, are printed on Mylar glued to the panels. The strip foils are glued on the Aramid panels with a precision better than $500 \mu \mathrm{m}$. The $3 \mathrm{~m}$ long wires are glued to support rods at three points to keep them at a constant $4 \mathrm{~mm}$ distance from the cathode planes and decrease the electrostatic and gravitational displacements of these wires. The gap is maintained at $8 \mathrm{~mm}$ using nine spacers facing melamine inserts embedded in the honeycomb structure of the panels. The potential wires are held at $-3200 \mathrm{~V}$ and the anode wires at $+1750 \mathrm{~V}$. The potentials on the strips provide a drift field of $1 \mathrm{kV} / \mathrm{cm}$. With this electric field and the gas mixture used, the ionization electrons drift with a velocity of about $50 \mathrm{~mm} / \mu \mathrm{s}$. In order to compensate for the Lorentz angle and to keep a drift direction parallel to the planes when the magnetic field is turned on, the potentials on the strips are set at different values on the two sides of each gap.

There are 49 chambers in the complete detector corresponding to 147 sense wire planes and a total of 6174 wires. The target chambers are mounted in 11 modules of four chambers each in the front part of the detector. Five additional chambers are installed individually in the TRD region and are used to improve the lever arm for tracking and for a better extrapolation of the tracks to the rest of the subdetectors.

Each chamber contributes 0.02 radiation length. The total fiducial mass of the chambers is 2.7 tons over an area of $2.6 \times 2.6 \mathrm{~m}^{2}$.

The chambers are operated at a voltage $100 \mathrm{~V}$ above the beginning of the plateau, which was found to be $200 \mathrm{~V}$ wide. Under these conditions, the typical wire efficiency is $97 \%$, most of the loss being due to the supporting rods. Wire signals are fed to a preamplifier and a fast discriminator, allowing track separation down to $1 \mathrm{~mm}$. Discriminator output signals are fed to Lecroy 1876 TDCs described above.

Space resolution has been studied using straight tracks (muons) crossing the detector during data taking. The distribution of residuals has been obtained after a careful alignment of all wires (fitted in several segments along the wires to take mechanical defects and electrostatic effects into account) as well as a detailed description of the time-to-distance relation. The distribution (Fig. 8) has a sigma of about $150 \mu \mathrm{m}$. The dependence of this quantity on drift distance and polar angle is shown in Fig. 9. The $5^{\circ}$ stereo angles give a resolution along the wires of $1.5 \mathrm{~mm}$.

The momentum resolution provided by the drift chambers is a function of momentum and track length. For charged hadrons and muons travelling normal to the plane of the chambers, it can be parametrized as:

$$
\frac{\sigma_{p}}{p} \approx \frac{0.05}{\sqrt{L}} \oplus \frac{0.008 p}{\sqrt{L^{5}}}
$$


where the momentum $p$ is in $\mathrm{GeV} / c$ and the track length $L$ in metres. The first term is the contribution from multiple scattering and the second term comes from the singlehit resolution of the chambers. For a momentum of $10 \mathrm{GeV} / c$, multiple scattering is the dominant contribution for track lengths longer than $1.3 \mathrm{~m}$.

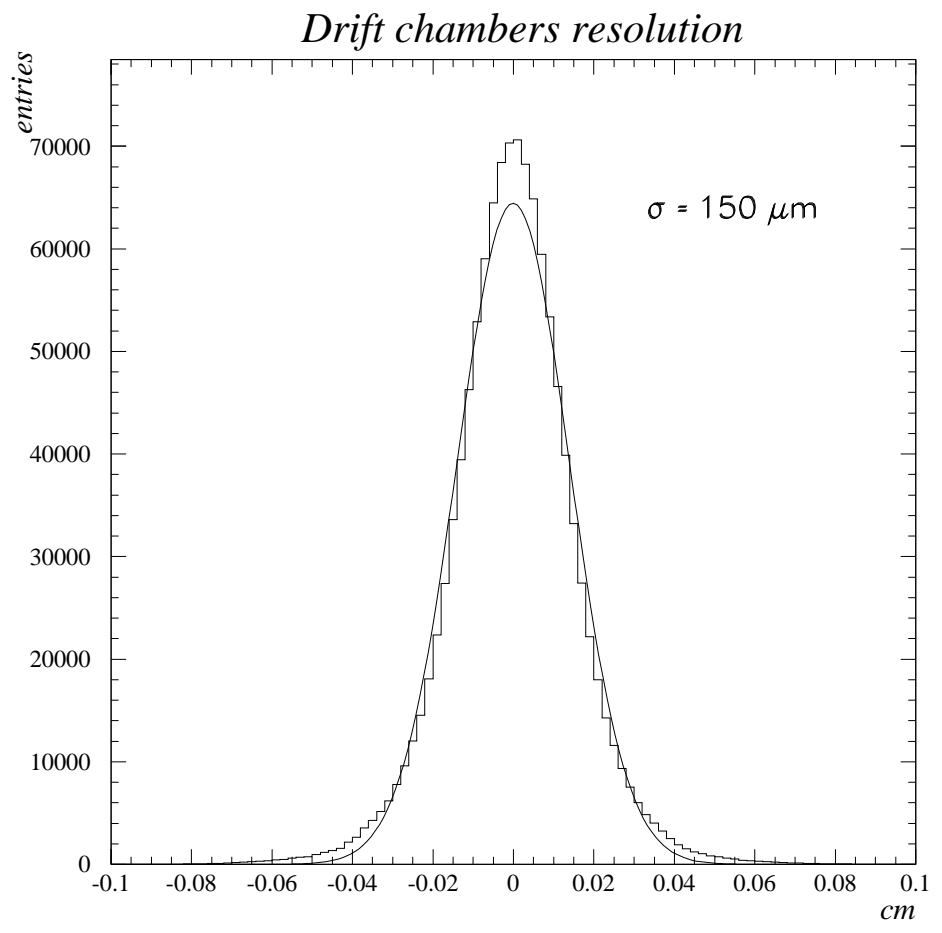

Figure 8: Residuals for a sample of straight tracks at normal incidence on the drift chambers

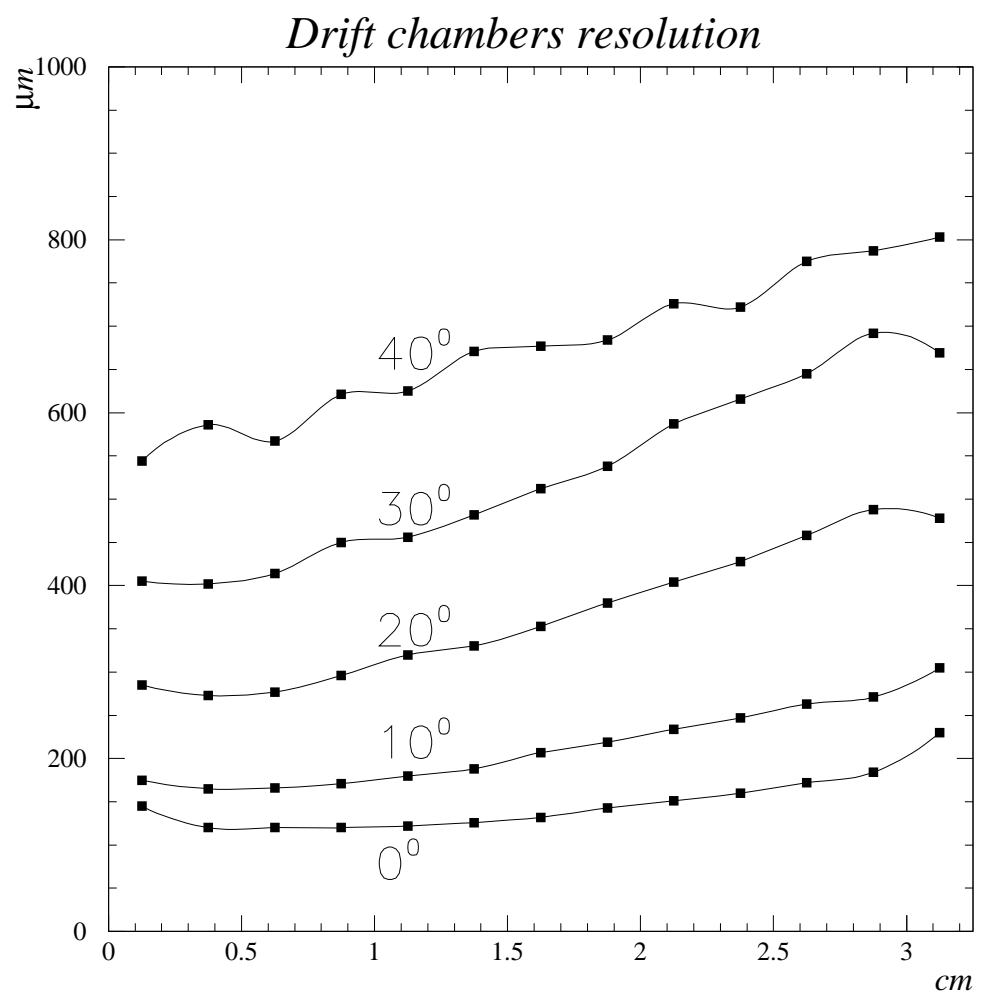

Figure 9: The dependence of the track residuals on drift distance and angle 
Table 2 shows the resolution for some typical values of $p$ and $L$.

The excellent track reconstruction obtained is demonstrated in Section 3.1 where the observed $\mathrm{K}_{\mathrm{s}}^{0}$ signal is presented.

The tracking is more difficult for electrons as they radiate photons via the bremsstrahlung process as they traverse the non-zero-density tracking system. This results in a continuously changing curvature. In this case, the resolution is worse and electron energies are measured by combining information from the drift chambers and the electromagnetic calorimeter.

Table 2: Resolutions obtained in the NOMAD drift chambers, for different track lengths and momenta

\begin{tabular}{|c|c|c|}
\hline$p[\mathrm{GeV} / c]$ & $L[\mathrm{~m}]$ & $\sigma_{p} / p[\%]$ \\
\hline 1.0 & 1.5 & 4.1 \\
& 3.5 & 2.7 \\
\hline 10.0 & 1.5 & 5.0 \\
& 3.5 & 2.7 \\
\hline 50.0 & 1.5 & 15.1 \\
& 3.5 & 3.2 \\
\hline
\end{tabular}

Most neutrino interactions in the NOMAD active target occur in the passive panels of the drift chambers. Interaction vertices are reconstructed by extrapolating the tracks of charged secondary-interaction products measured in the chambers. Figure 10 shows the distribution of vertices in the plane perpendicular to the beam. A fiducial cut of $-120 \leq x, y \leq 120 \mathrm{~cm}$ is imposed. The gradual diminishing of the beam intensity with radius is easily discernible. The nine square spots of high intensity are caused by the melamine inserts which are built into the chambers in order to maintain the $8 \mathrm{~mm}$ gap widths.

Figure 11 shows the distribution of vertices along the beam direction. The information from the 44 drift chambers has been overlayed to cover the $\approx \pm 5 \mathrm{~cm}$ about the centre of each chamber. The eight 'spikes' in this distribution correspond to the Kevlar skins of the drift chambers, see Fig. 7. Regions in $z$ with a low interaction rate correspond to the three gas-filled drift gaps and the honeycomb panels.

A much more detailed description of these chambers will be given in a forthcoming publication [16].

\subsection{Trigger counters}

Two trigger planes are installed in the NOMAD detector: the first plane follows the active target and the second plane is positioned behind the TRD region. Each of them covers a fiducial area of $280 \times 286 \mathrm{~cm}^{2}$ and consists of 32 scintillation counters with singleended photomultiplier readout. The scintillators have a thickness of $0.5 \mathrm{~cm}$ and a width of $19.9 \mathrm{~cm}$. Twenty-eight of the counters are installed horizontally and have a length of $124 \mathrm{~cm}$. In order to increase the fiducial area of the trigger planes, four counters of $130 \mathrm{~cm}$ length are installed vertically to cover the light guides of the horizontal counters. 


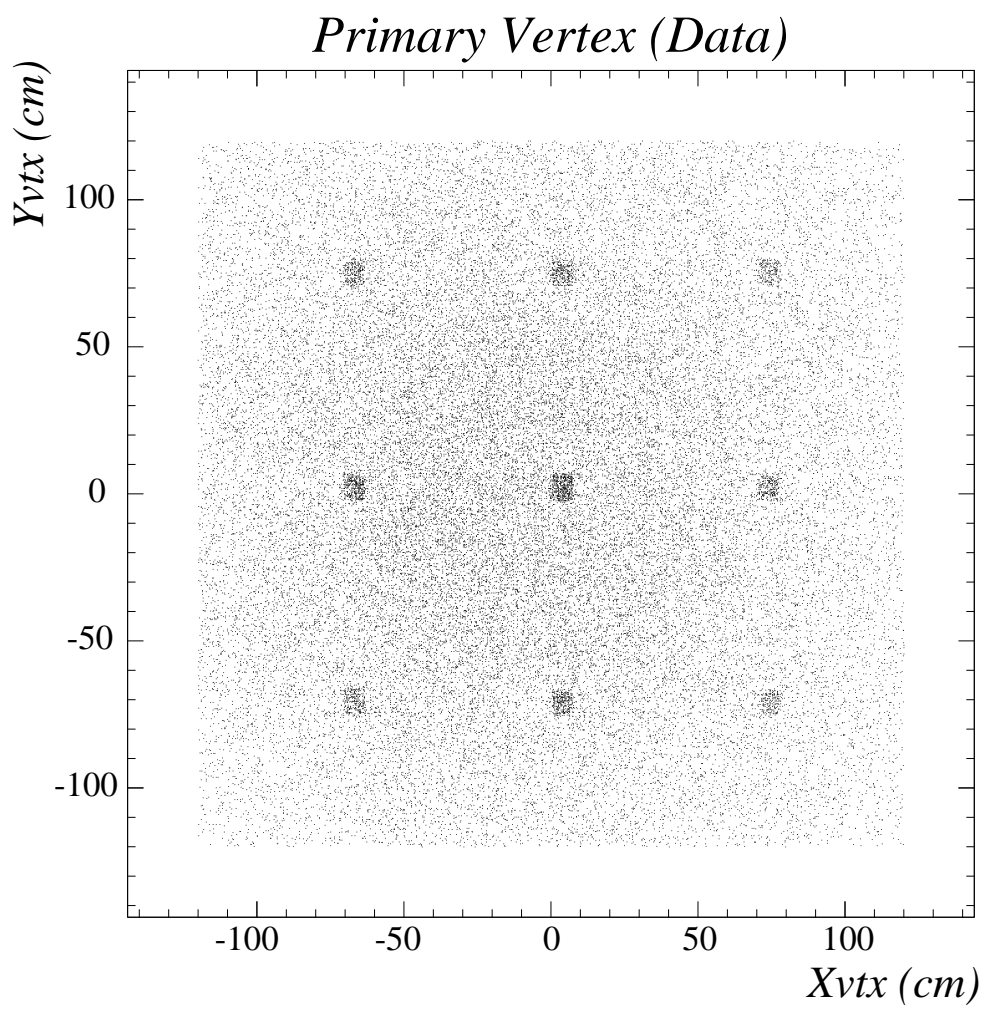

Figure 10: The positions of reconstructed neutrino interaction vertices in the plane perpendicular to the beam; see text.

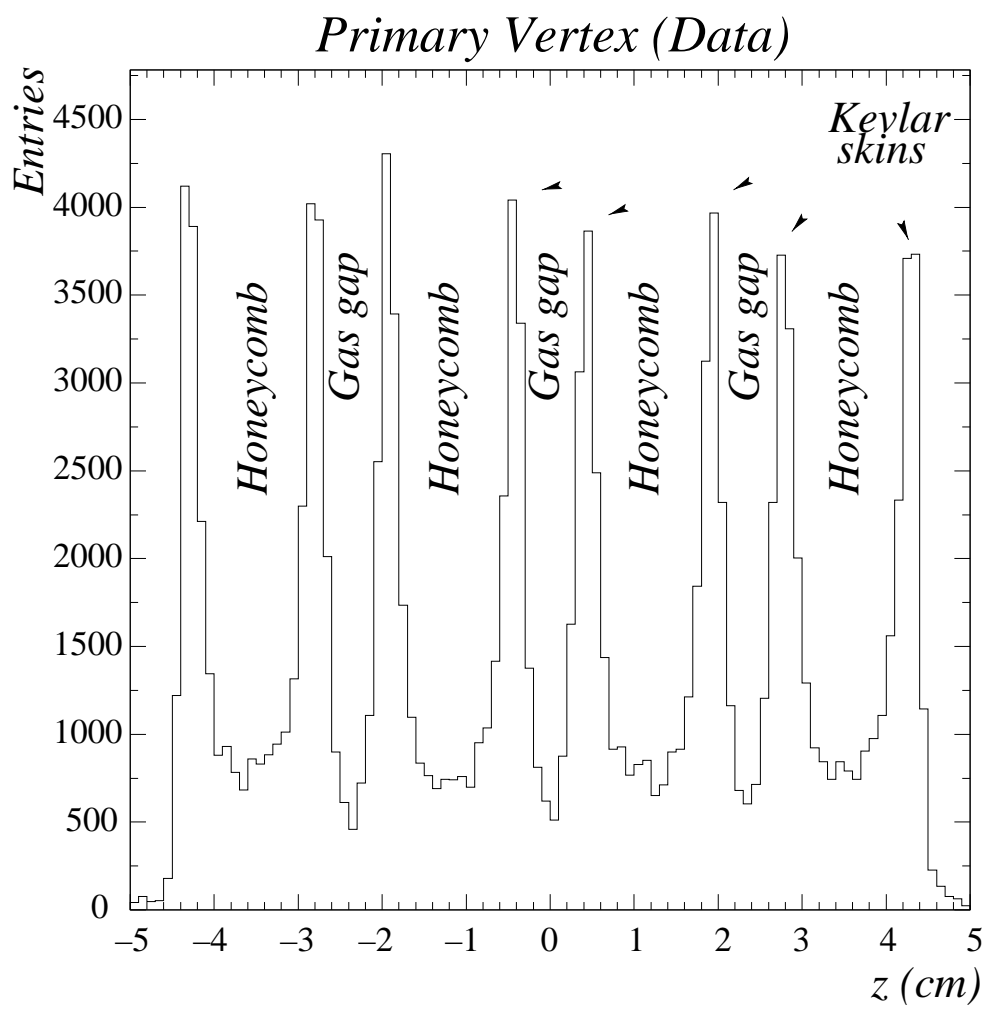

Figure 11: The positions of reconstructed neutrino interaction vertices as measured along the beam direction; see text. 
The scintillators are connected by adiabatic light guides to 16-dynode photomultipliers of the proximity mesh type [17], which are oriented parallel to the magnetic field. The field of $0.4 \mathrm{~T}$ reduces the response of these photomultipliers by only $30 \%$. They have an intrinsic time resolution of $1 \mathrm{~ns}$ and a noise rate of less than $50 \mathrm{~Hz}$. The discriminated photomultiplier signals are input into a trigger module (LeCroy 4564) where a logical OR is performed for each plane. A coincidence between the two planes is required for a valid trigger. The discriminated photomultiplier signals are also sent to a TDC (LeCroy 1876 Module 100), which allows the individual counter time to be determined offline.

The average efficiency of the trigger counters, for single tracks, has been determined with data and found to be $97.5 \pm 0.1 \%$.

\subsection{Transition Radiation Detector}

The NOMAD Transition Radiation Detector (TRD) has been designed to separate electrons from pions with a pion rejection factor greater than $10^{3}$ for a $90 \%$ electron efficiency in the momentum range from $1 \mathrm{GeV} / c$ to $50 \mathrm{GeV} / c$. This factor, together with the additional rejection provided by the preshower and electromagnetic calorimeter, is needed in the oscillation search in the $\tau^{-} \rightarrow \mathrm{e}^{-} \nu_{\tau} \bar{\nu}_{\mathrm{e}}$ decay channel in order to eliminate neutral current neutrino interaction events in which an isolated pion track fakes an electron.

The large rejection factor required and the large lateral dimensions of the detector $\left(2.85 \times 2.85 \mathrm{~m}^{2}\right)$ make the NOMAD TRD one of the largest transition radiation detectors ever built. Its design was optimized by detailed simulation and after several test beam measurements $[18,19]$. It takes into account two main experimental constraints: the limited longitudinal space inside the NOMAD magnet and the requirement that there be less than $2 \%$ of a radiation length added between two consecutive measurements in the drift chambers.

The TRD is located after the first trigger plane and consists of nine identical modules. The first eight modules are paired into four doublets. In order to provide a precise track extrapolation from the drift chamber target to the calorimeter, five drift chambers are embedded in the TRD, one after each TRD doublet and one after the last module, see Figs. 1 and 2. Each TRD module includes a radiator followed by a detection plane with the following design (see Fig. 12):

- $\quad$ The radiator is a set of 315 polypropylene foils, each $15 \mu \mathrm{m}$ thick and $2.85 \times 2.85 \mathrm{~m}^{2}$ in area, separated by $250 \mu \mathrm{m}$ air gaps. The foils are stretched on an aluminium frame and embossed to ensure a regular spacing in spite of their large size and of electrostatic effects.

- The detection plane consists of 176 vertical straw tubes, each $3 \mathrm{~m}$ long and $16 \mathrm{~mm}$ in diameter, separated by $0.2 \mathrm{~mm}$. The straw tubes are fed in parallel with a $80 \%$ xenon - $20 \%$ methane gas mixture. They are made of two shifted $12.5 \mu \mathrm{m}$ thick ribbons of aluminized (115 nm of aluminium) Mylar rolled and glued along a $16 \mathrm{~mm}$ diameter helix. The sensitive anode is a $50 \mu \mathrm{m}$ diameter gold-plated tungsten wire stretched with a tension of $100 \mathrm{~g}$. In order to protect the gas mixture in the straw tubes from water and oxygen contamination, each TRD module is covered by two aluminized Mylar skins with a permanent flow of dry $\mathrm{N}_{2}$ between them. 


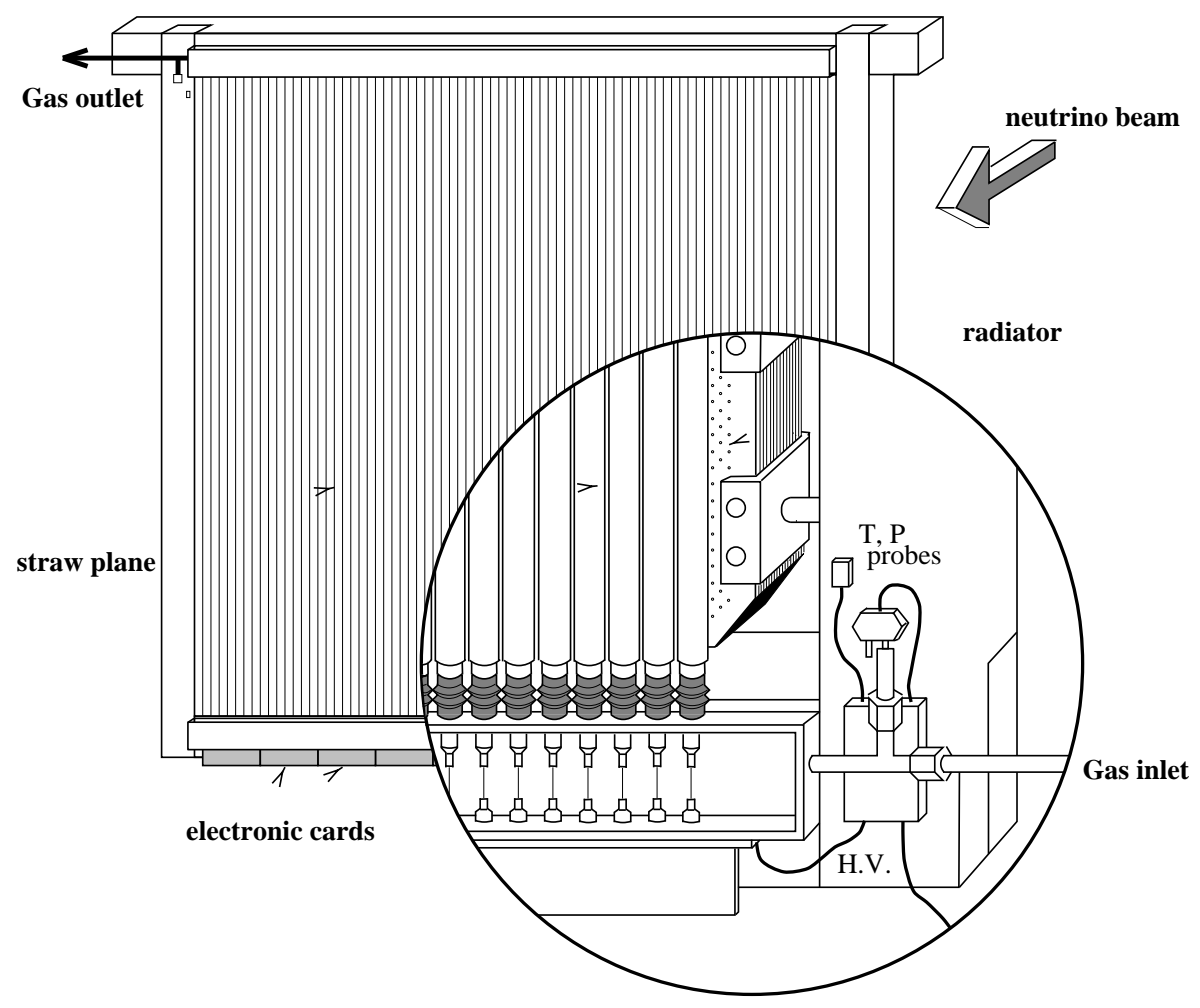

Figure 12: Schematic view of a TRD module, as seen from the straw plane side.

The signals from the 1584 straw tubes working in the proportional mode are fed into preamplifiers producing differential outputs. These signals are transmitted via 32 twisted-pair cables to differential drivers located outside the magnet. The charge ADCs are read out by a VME system. A test pulse system is used to control the stability of the entire electronic chain.

Continuous calibration of the TRD is performed with the help of ${ }^{55}$ Fe radioactive sources $\left(E_{\gamma}=5.89 \mathrm{keV}\right)$ uniformly deposited on a ribbon stretched horizontally in the middle of each detection plane. A self-triggering system is used to record the ${ }^{55} \mathrm{Fe}$ signals in each straw between every neutrino spill. The signals of the ${ }^{55} \mathrm{Fe}$ sources reflect all of the changes in the detector conditions (high voltage, temperature, pressure, gas mixture composition, etc.), thus allowing efficient monitoring and calibration of the TRD. In addition, most of the working parameters are permanently controlled by a specially developed 'slow control' system.

Electron identification in the TRD is based on the difference in the total energies deposited in the straw tubes by particles of different Lorentz factors $\left(\gamma=E / m c^{2}\right)$. Charged particles with $\gamma<500$ deposit energy in the straw tube predominantly by ionization losses, whereas charged particles with $\gamma>500$ (mainly electrons in NOMAD) also produce transition radiation X-rays at the interfaces of the foils [20]. As a result, a few photons in the kiloelectron volt range are produced by an electron crossing a radiator $\left(\langle N\rangle=3.1\right.$ with $\langle E\rangle=14 \mathrm{keV}$ for $\left.10 \mathrm{GeV} / c \mathrm{e}^{-}\right)$. About $60 \%$ of the photons emitted from the radiator are absorbed in the detection planes, due to the large cross-section of xenon for photons of a few kiloelectron volts. Transition radiation X-ray energy deposition is added to the ionization losses of the parent particle in the same straw tube, because the angular distribution of emitted photons peaks around the initial particle direction (the mean angle of emission is about $1 / \gamma)$. 
The algorithm developed for electron identification [21] is based on a likelihood ratio method and relies on test beam measurements and detector simulation. The TRD simulation has been extensively tested in situ using the muons $\left(5 \mathrm{GeV} / c<p_{\mu}<50 \mathrm{GeV} / c\right)$ crossing the detector during the flat top between the two neutrino spills. Figure 13 shows a comparison between the experimental and simulated distributions of the energy deposited in straw tubes by $5 \mathrm{GeV} / c$ muons (ionization losses only) and by $\delta$-ray electrons with a mean momentum of about $2 \mathrm{GeV} / c$, emitted by muons (sum of ionization losses and detected transition-radiation photons).

A pion rejection factor greater than 1000 is obtained with the nine TRD modules in the momentum range from $1 \mathrm{GeV} / c$ to $50 \mathrm{GeV} / c$, while retaining an electron efficiency of $90 \%$ (see Section 3.4).

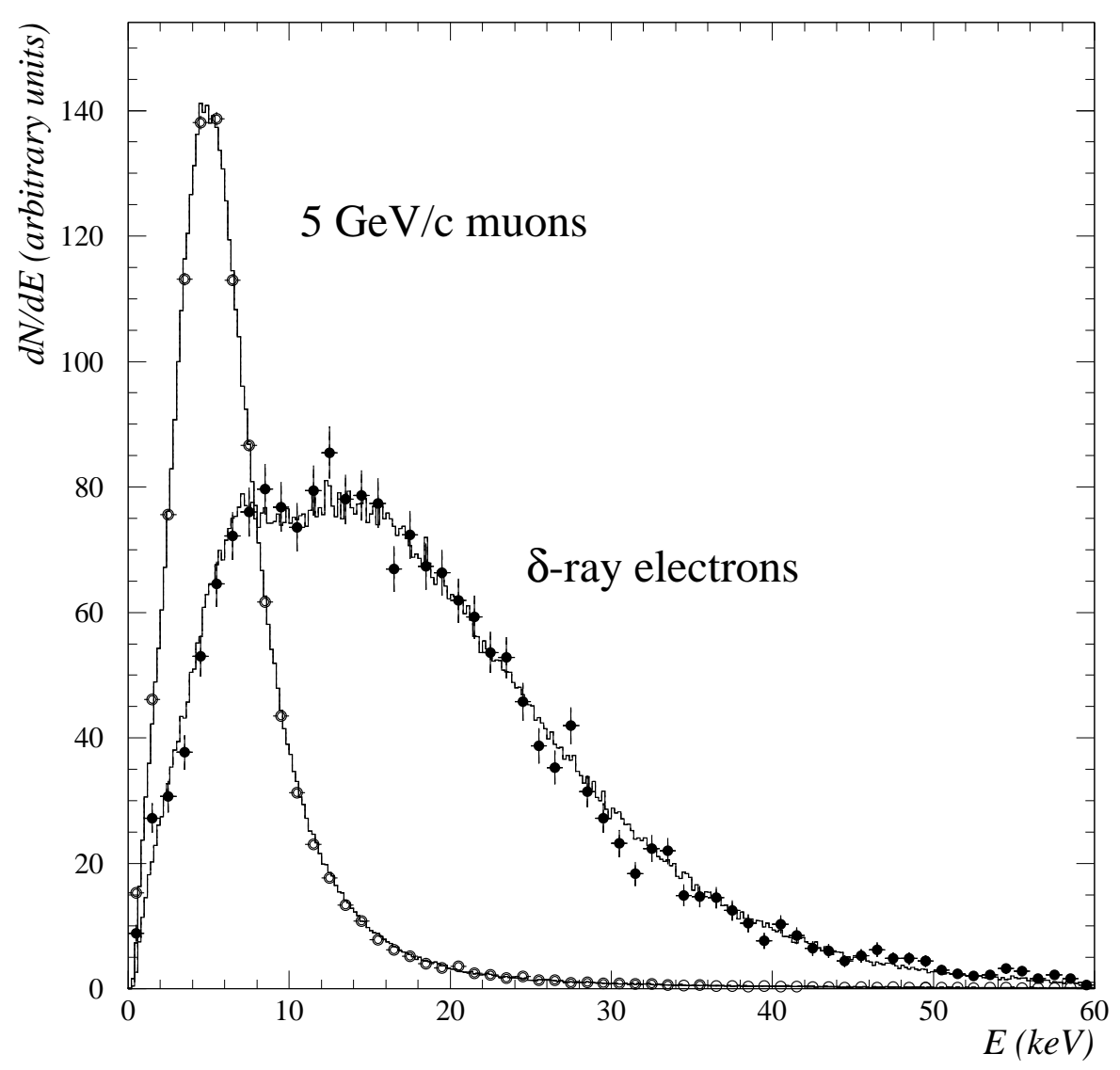

Figure 13: Comparison of experimental (points with error bars) and simulated (solid lines) distributions of the energy deposited in TRD straw tubes by $5 \mathrm{GeV} / c$ muons (open circles) and $2 \mathrm{GeV} / c$ electrons (closed circles).

\subsection{Preshower detector}

The Preshower (PRS) detector, which is located just in front of the electromagnetic calorimeter, is composed of two planes of proportional tubes (286 horizontal and 288 vertical tubes $)$ preceded by a $9 \mathrm{~mm}\left(1.6 \mathrm{X}_{0}\right)$ lead-antimony $(96 \%-4 \%)$ converter, see Fig. 14.

The proportional tubes are made from extruded aluminium profiles and are glued to two aluminium end-plates of $0.5 \mathrm{~mm}$ thickness. Each tube has a square cross-section of $9 \times 9 \mathrm{~mm}^{2}$ and the walls are $1 \mathrm{~mm}$ thick. The $30 \mu \mathrm{m}$ gold-plated tungsten anode is strung with a tension of $50 \mathrm{~g}$ and secured at each end in hollow copper pins. In order to avoid wire vibrations, the anodes are also glued in the middle of the preshower on small 
resofil spacers. The proportional tubes operate at a voltage of $1500 \mathrm{~V}$, with a mixture of (80:20) $\mathrm{Ar}: \mathrm{CO}_{2}$.

Signals from each tube are fed into charge preamplifiers; at the output of the preamplifier, two pulses of opposite polarity are sent to the remote Delaying and Pulse Shaping Amplifier (DPSA) via twisted-pair cables. The use of symmetric drivers and differential receivers allows low-loss transmission along the twisted-pair bundles. The twelve preamplifier signals are sent to the ADC via a 270 ns delay line.

\section{Assembly of the NOMAD Preshower}

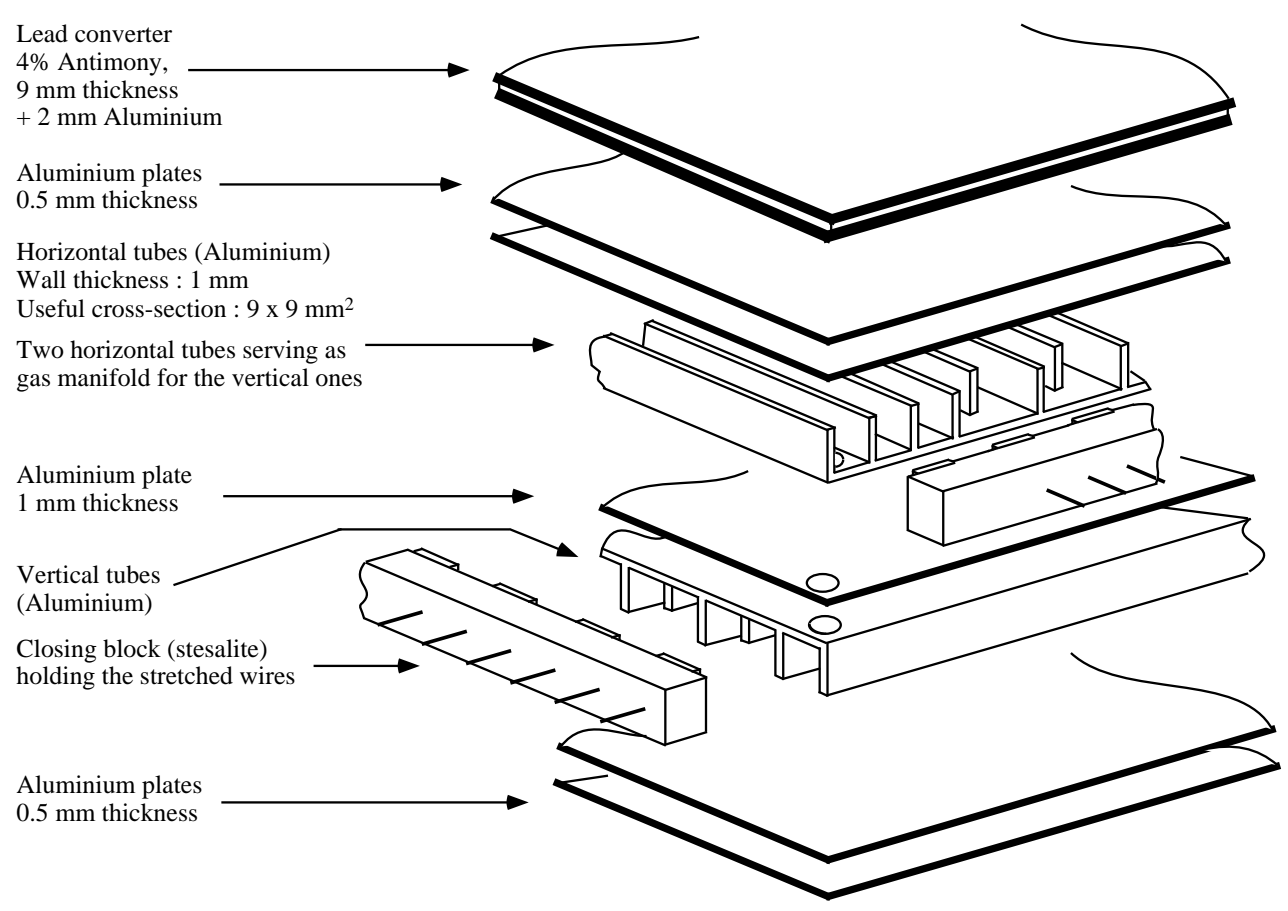

Figure 14: An exploded view of the Preshower

Large samples of straight-through muons are collected during the flat top of the SPS cycle. These muons have been used to monitor the preshower gains against pressure and temperature variations. More than 300000 such events, collected over about a month, have been analysed. Such high statistics are needed to make good fits to the distributions of energy deposition in each tube. Global variations of the gain induced by pressure and temperature fluctuations can nevertheless be calculated on a run-by-run basis. The electronic gains for each channel are then taken into account, allowing the final determination of the individual gas gain. The dispersion of these gas gains is found to be $6 \%$ for the 574 tubes, resulting mainly from inhomogeneity of gas flow and measurement errors.

The fine granularity of the PRS also assists in the understanding of signals in the calorimeter blocks caused by adjacent particles. Once the clusters with an associated track have been removed, the remaining ones might be attributed to photons which have converted in the PRS. The spatial resolution of the PRS for photons has not been directly measured. We estimate the r.m.s. resolution for a photon hitting the PRS using negative pions from the test beam interacting in the lead of the PRS. We make the assumption that these interactions are mainly charge exchange with one of the photons from the $\pi^{0}$ converting in the lead plate. The r.m.s. spatial resolution for these events is $1 \mathrm{~cm}$, a value which is much smaller than the dimensions of a single tower of the electromagnetic calorimeter and which allows the use of the PRS to determine the impact point of con- 
verting photons for the $70 \%$ of the photons which convert in the lead radiator. Using the above value as spatial resolution of photons and the energy resolution of the electromagnetic calorimeter, a rough estimation of the $\pi^{0}$ mass resolution is found to be $11 \mathrm{MeV} / c^{2}$, in good agreement with the observed $\pi^{0}$ mass distribution, see Section 3.2.

\subsection{Electromagnetic calorimeter}

The search for $\nu_{\tau}$ events in NOMAD relies strongly on electron identification as well as on a very accurate determination of the total transverse momentum in the event.

While electron identification is performed using the Transition Radiation Detector (TRD), the Electromagnetic Calorimeter (ECAL) is crucial for accurate measurement of electron and gamma energies from $100 \mathrm{MeV}$ up to $100 \mathrm{GeV}$ and for the determination of the neutral component of the transverse momentum. The large energy range to be covered requires a large dynamic range in the response of the detector and of the associated electronics. A lead-glass detector was chosen for its excellent energy resolution and uniformity of response. In NOMAD the electromagnetic calorimeter combined with the Preshower Detector (PRS) was also used to help improve the electron identification provided by the TRD.

The ECAL consists of 875 lead-glass Cerenkov counters of TF1-000 type arranged in a matrix of 35 rows and 25 columns. Each counter is a 19 radiation lengths deep block with a rectangular cross section of $79 \times 112 \mathrm{~mm}^{2}$. The direction of the $B=0.4$ T magnetic field, perpendicular to the counter axis, imposes severe constraints on the mechanical assembly of the light detection system (Fig. 15). The light detectors (two-stage photomultipliers, tetrodes, with a typical gain of 40 in the operating conditions of NOMAD) are coupled to the back face of the lead-glass blocks. This face is cut at $45^{\circ}$ with respect to the block axis, in such a way that the symmetry axis of the tetrodes forms an angle of $45^{\circ}$ with respect to the field direction, thus keeping the signal reduction caused by the magnetic field to less than $20 \%$. This reduction was found to be very constant and uniform. A low-noise electronic chain, composed of a charge preamplifier followed by a shaper and a peak sensing ADC with a resolution of 12 bits, provides a calorimeter response in a dynamic range larger than $4 \times 10^{3}$.

A fast analog signal from each shaper, discriminated and ORed over groups of 16 adjacent counters, is also provided for time measurements in order to reject energy depositions not associated with the triggered event. The obtained time resolution is a few nanoseconds for energy depositions larger than $1 \mathrm{GeV}$. In addition the fast analog signals, summed over groups of 64 counters, are discriminated and ORed over all the groups to form an ECAL trigger, as explained in Section 2.11.

Monitoring of the lead-glass response is performed using two blue Light Emitting Diodes (LED) per counter mounted on the same face of the block on which the tetrodes are positioned; a detailed description of this monitoring system is given elsewhere [22]. The LEDs are driven by highly stable current pulses, generated by specially designed current drivers. This system has been shown to be very stable over periods of several months as seen in Fig. 16(a) and (b) where the ratio of the LED signals to a reference measurement and its spread are shown. 


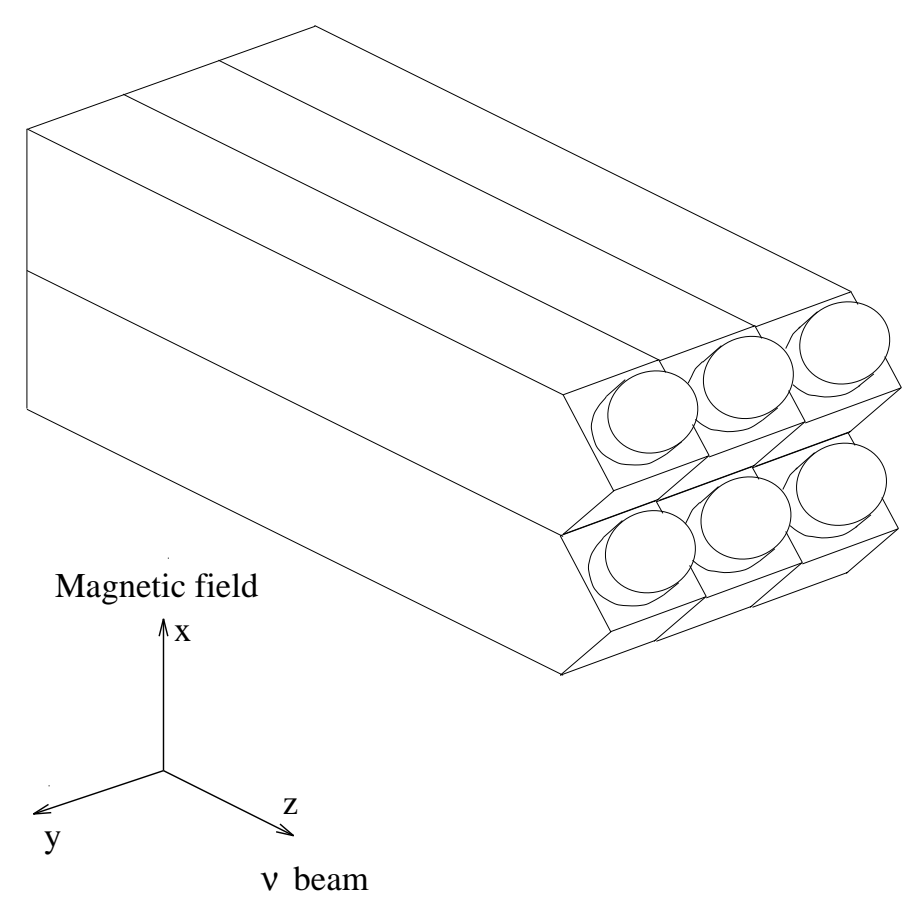

Figure 15: Schematic view of some lead-glass counters with phototetrodes
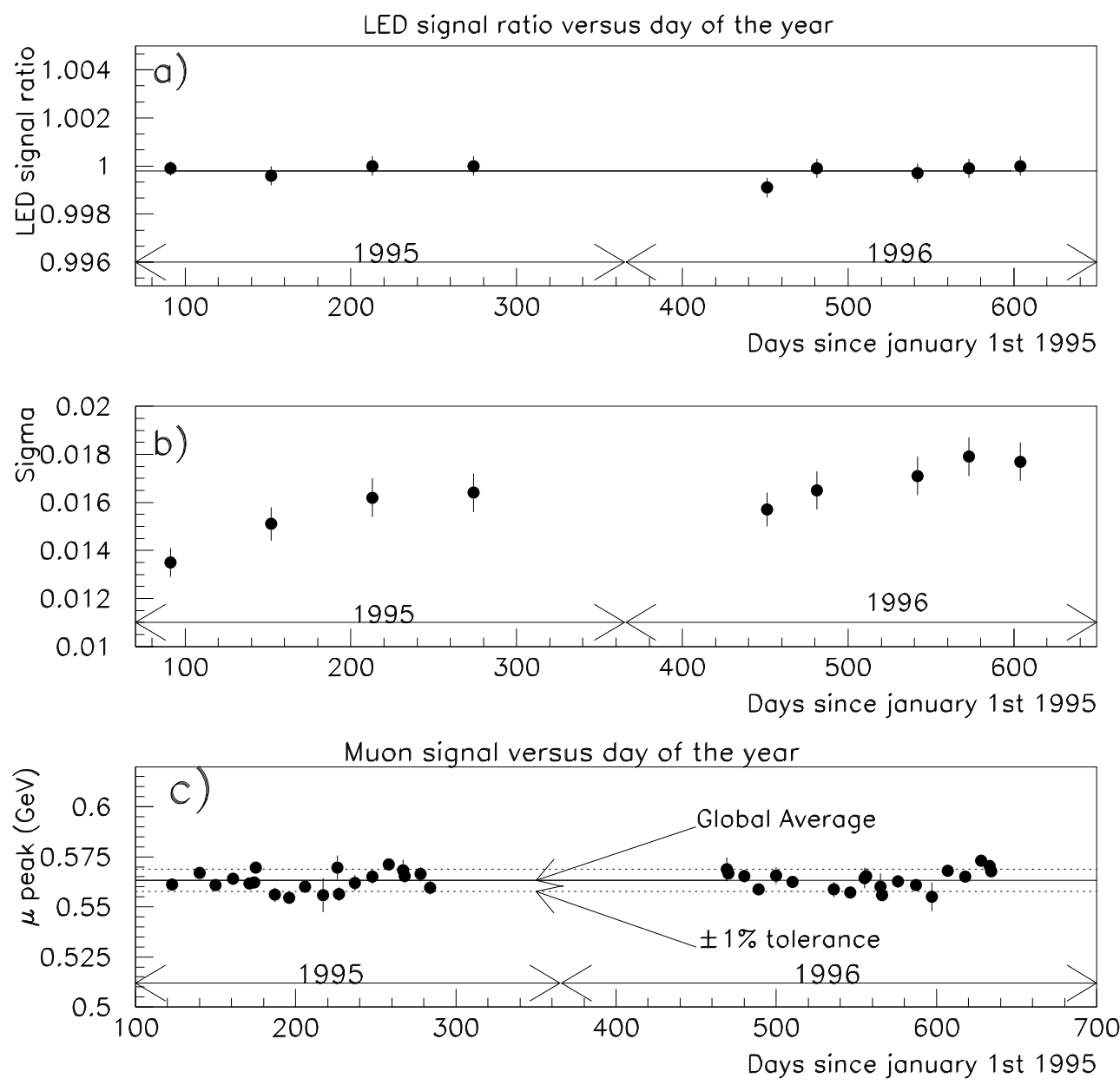

Figure 16: The signal in ECAL from LED and muon monitoring: (a) LED stability in time over two years of data collection, (b) spread of the ratio of the LED signals to a reference measurement, (c) $\mu$ peak position vs. time over two years of data collection. 
Before the final assembly in NOMAD, the lead-glass blocks were individually calibrated using a $10 \mathrm{GeV} / c$ electron beam having a small momentum spread $(\Delta p / p=1 \%)$. The response of each block to the LED pulses, measured immediately after the corresponding electron calibration, was also recorded and used to convert to the NOMAD operating conditions the calibration obtained at the test beam in the absence of a magnetic field. The effect of the magnetic field on the calibration was taken into account by LED measurements performed with and without magnetic field.

The linearity of the calorimeter response to electrons was verified at the test beam in the energy range 1.5-80 GeV. Deviations from linearity are kept below $1.0 \%$ by applying a logarithmic correction to the cluster energy as explained in Ref. [23].

A detailed study of the energy resolution was performed. After the deconvolution of the electronic noise from the data, a two-parameter fit of the energy resolution $\Delta E / E=$ $a+b / \sqrt{ } E$, where $E$ is in $\mathrm{GeV}$, gives $a=(1.04 \pm 0.01) \%$ and $b=(3.22 \pm 0.07) \%$.

The ECAL response as a function of the impact point of the incoming electrons has been measured to be uniform within $\pm 0.5 \%$; the response to electrons entering at normal incidence in the gaps between adjacent blocks has shown that ECAL is 'hermetic' at more than $99 \%$. A weak dependence of the total energy release on the angle of incidence $\theta$ of the incoming electrons has been found both in the test beam measurements and in the Monte Carlo simulations: this dependence, which has been empirically parametrized as $E(\theta)=E \sqrt{ } \cos (\theta)$, can be attributed to the small variation with angle of the Cerenkov light collection efficiency. In addition, the number of towers receiving contributions from a given shower increases with angle. However, this does not affect the overall energy resolution that remains insensitive to both shower position and angle. The shower position has been measured with an average resolution of about $4 \mathrm{~mm}$ in each direction $(6 \mathrm{~mm}$ at the centre of the block and $2 \mathrm{~mm}$ at the edges).

The calibration and the calorimeter response to low-energy photons have been checked by measuring the $\pi^{0}$ mass both in a dedicated test-beam and during the NOMAD data taking. The following results were found using test beam data:

$$
m_{\pi}=(133.7 \pm 1.2) \mathrm{MeV} / c^{2} \quad, \quad \sigma_{m}=16 \mathrm{MeV} / c^{2} .
$$

The analogous results obtained during the normal NOMAD data taking are discussed in Section 3.2.

The calorimeter response to muons corresponds to a peak value for the energy deposition of $(0.566 \pm 0.003) \mathrm{GeV}$, as shown in Fig. 17. The muon signals were found to be stable within $\pm 1 \%$ over a two-year period providing an independent check of the stability in calorimeter response; this is complementary to the one provided by the LED monitoring that is continuously running during normal data-taking. Figure 16(c) shows the average energy deposited by muons crossing ECAL as a function of time.

Triggers based on the ECAL energy deposition are currently being acquired, together with the 'normal' neutrino triggers described elsewhere. The large mass of ECAL $(\approx 20 \mathrm{t})$ gives a trigger rate of $\approx 2.5$ interactions/neutrino spill. The large acceptance makes such events very useful for physics purposes as well as for monitoring the beam conditions.

A more detailed description of ECAL is given elsewhere [24]. The performance of ECAL when exposed to test beams of electrons, pions, and muons is reported in Ref. [23]. 


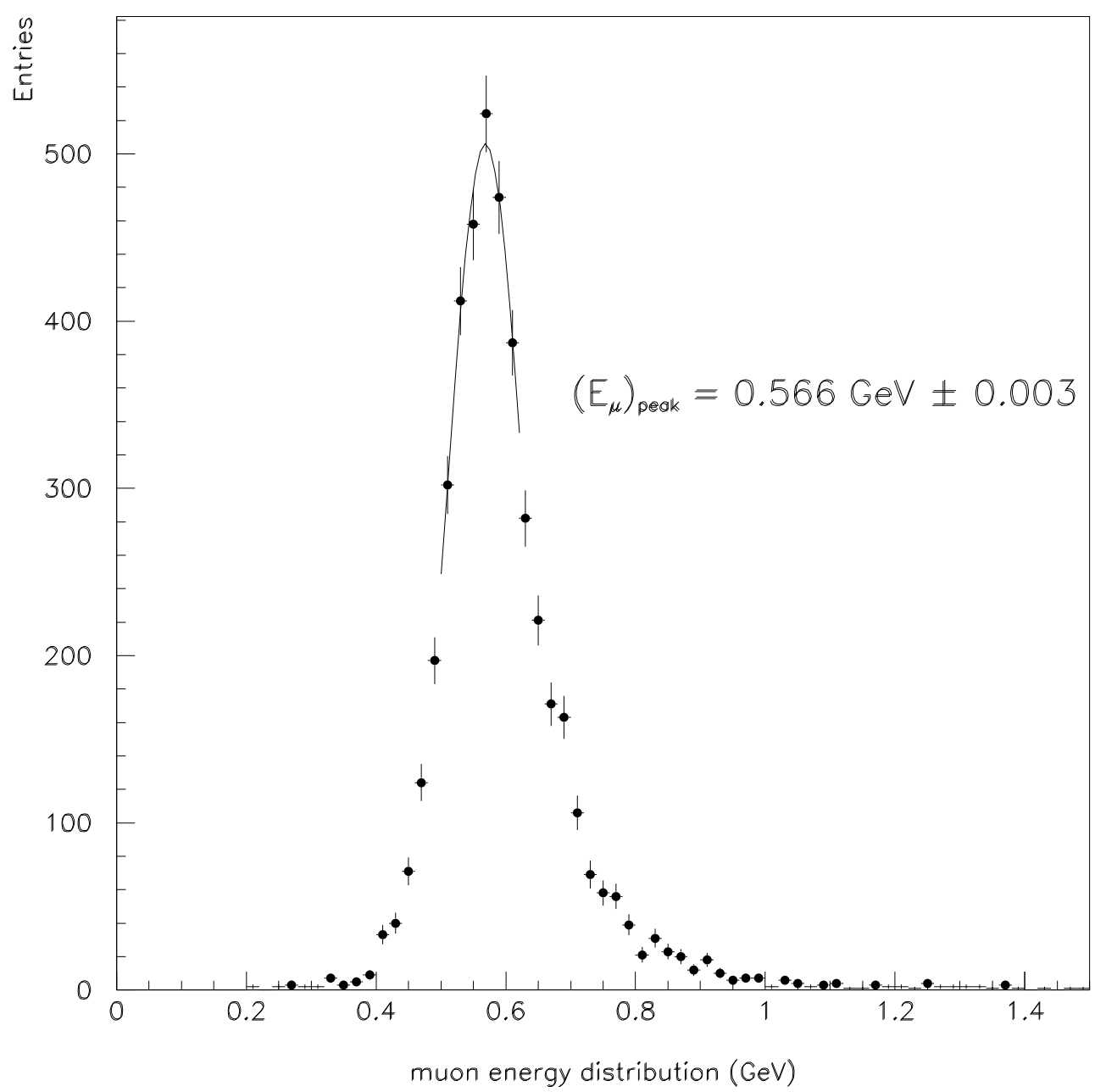

Figure 17: The signal in ECAL from muons crossing the detector during normal data taking. A correction has been applied to account for the differences in impact angle of the muons on the detector.

\subsection{Hadronic calorimeter}

The Hadron Calorimeter (HCAL) is intended to detect neutral hadrons and to provide a measurement of the energy of charged hadrons complementary to that derived from momentum measurements in the drift chambers. Knowledge of neutral hadrons is important when constructing kinematic quantities such as missing transverse momentum, and calorimetric measurements of charged particles can be used both as a consistency check on the momentum measurement of the charged particles and as an aid in distinguishing between muons and charged hadrons.

The HCAL is an iron-scintillator sampling calorimeter. The NOMAD detector is suspended from iron pillars (the I's) at the two ends of the magnet. The downstream pillar was instrumented with scintillators to construct the HCAL. The I's consist of 23 iron plates, $4.9 \mathrm{~cm}$ thick, separated by $1.8 \mathrm{~cm}$ gaps, and six of these modules form a wall $5.4 \mathrm{~m}$ wide, $5.8 \mathrm{~m}$ high, and $1.5 \mathrm{~m}$ deep. This wall acts as a filter for the large muon chambers downstream and as a support for the 'basket' which contains much of the NOMAD detector.

The active elements of the calorimeter are scintillator paddles $3.6 \mathrm{~m} \mathrm{long,} 1 \mathrm{~cm}$ thick, and (on average) $18.3 \mathrm{~cm}$ wide. Tapered acrylic light pipes are glued to each end of the scintillator paddle to form an assembly $5.52 \mathrm{~m}$ long. Eleven of these assemblies 
are threaded horizontally through the first 11 gaps in the iron wall to form a calorimeter module $18.3 \mathrm{~cm}$ high, and approximately 3.1 interaction lengths $\left(\lambda_{\text {int }}\right)$ deep. Scintillation light is directed through adiabatic light guides to a 5 inch phototube at each end of the module. Eighteen of these modules are stacked vertically to form a calorimeter with an active area $3.6 \mathrm{~m}$ wide by $3.5 \mathrm{~m}$ high. A schematic front view of the HCAL, showing the scintillators and tapered acrylic light pipes, is given in Fig. 18.

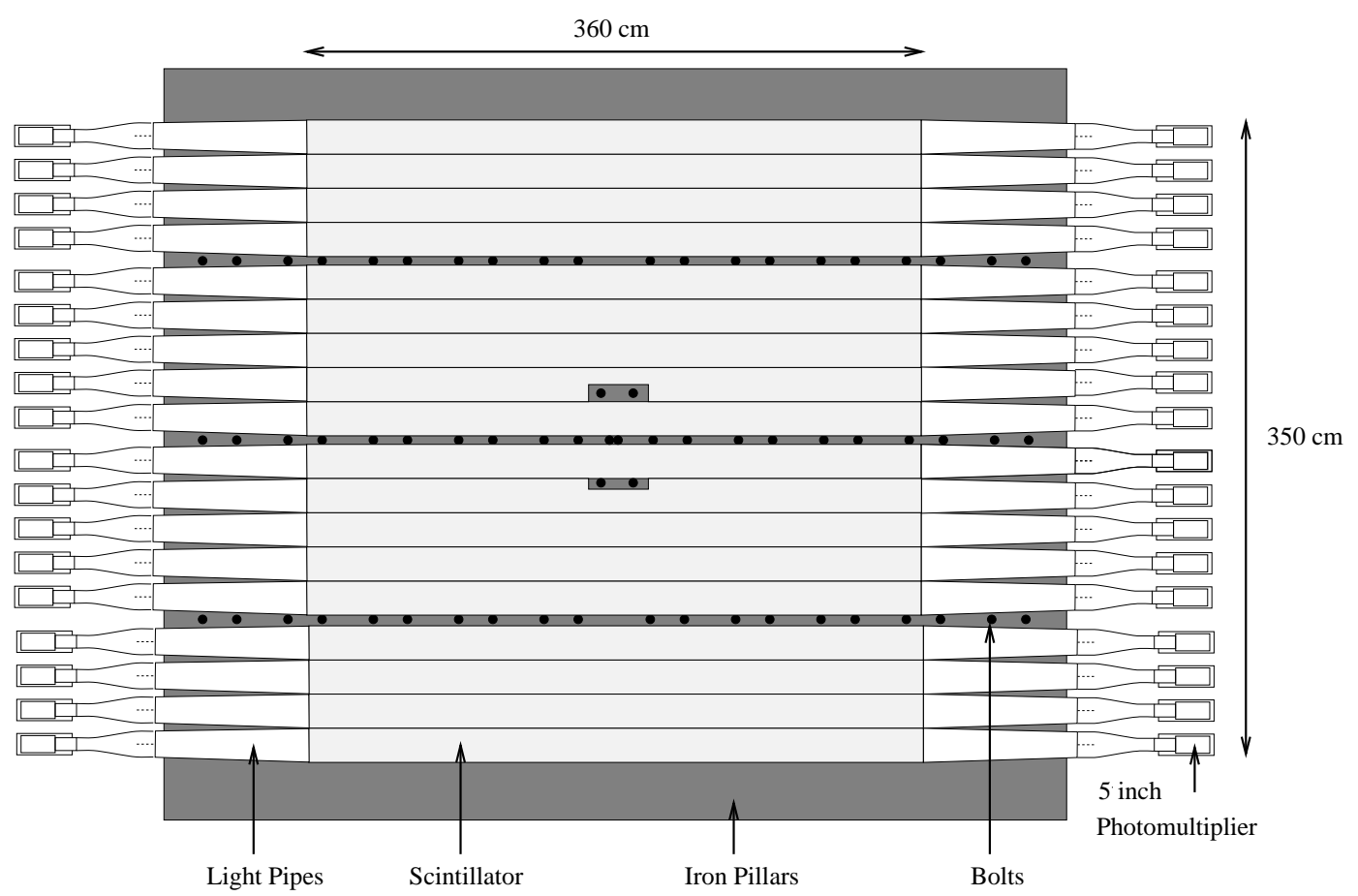

Figure 18: A front view of the HCAL; see the text for details.

The output from each phototube is split. One signal is delayed and sent to a chargeintegrating ADC, while the second is discriminated and sent to a multihit TDC. The ADC signals are used for energy and position measurements, while the TDC signals are used to determine event timing. The discriminated signals can also be used, in conjunction with a majority logic circuit, to form a trigger.

The energy deposited in a given module is obtained from the geometric mean of the two phototube signals, and the horizontal position of the energy deposit is determined from the attenuation length of the scintillator and the ratio of the phototube signals. Vertical positions are determined from the pattern of energy sharing between the modules. Figure 19 shows the energy response to muons which traverse the calorimeter; the peak of the minimum-ionizing distribution is at $1.5 \mathrm{GeV}$ and the expected Landau shape is clearly seen. Figure 20 shows the difference between the predicted horizontal position and the position measured by the hadron calorimeter for muons passing through a single calorimeter module; typical position resolutions are of the order of $20 \mathrm{~cm}$.

There is a high probability that hadrons will begin to shower in the approximately $2.1 \lambda_{\text {int }}$ of material upstream of the hadron calorimeter, and so the total hadronic energy is taken to be a weighted sum of the energies deposited in the hadron and electromagnetic calorimeters. 


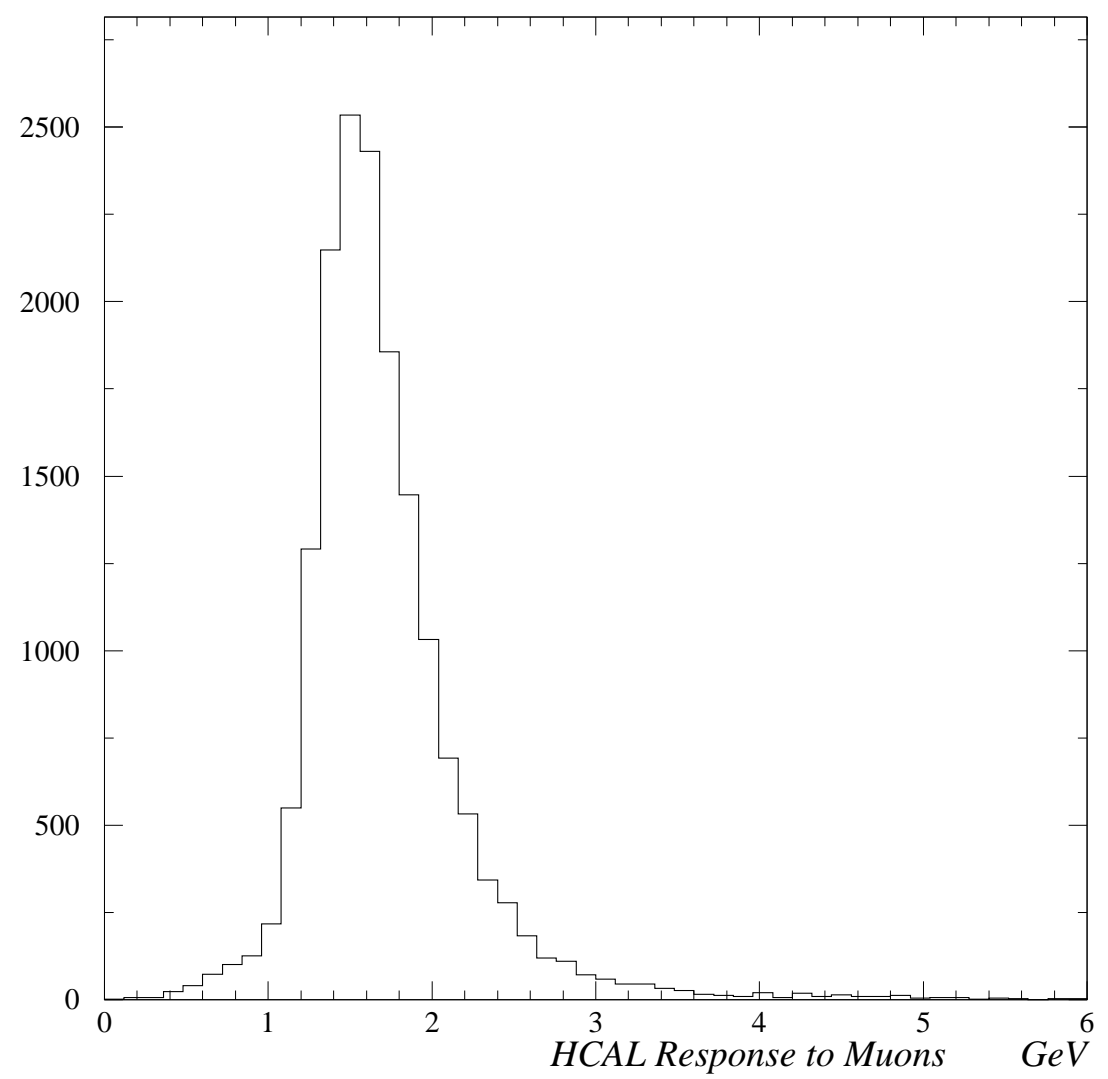

Figure 19: Minimum-ionizing peak for muons passing through the hadron calorimeter

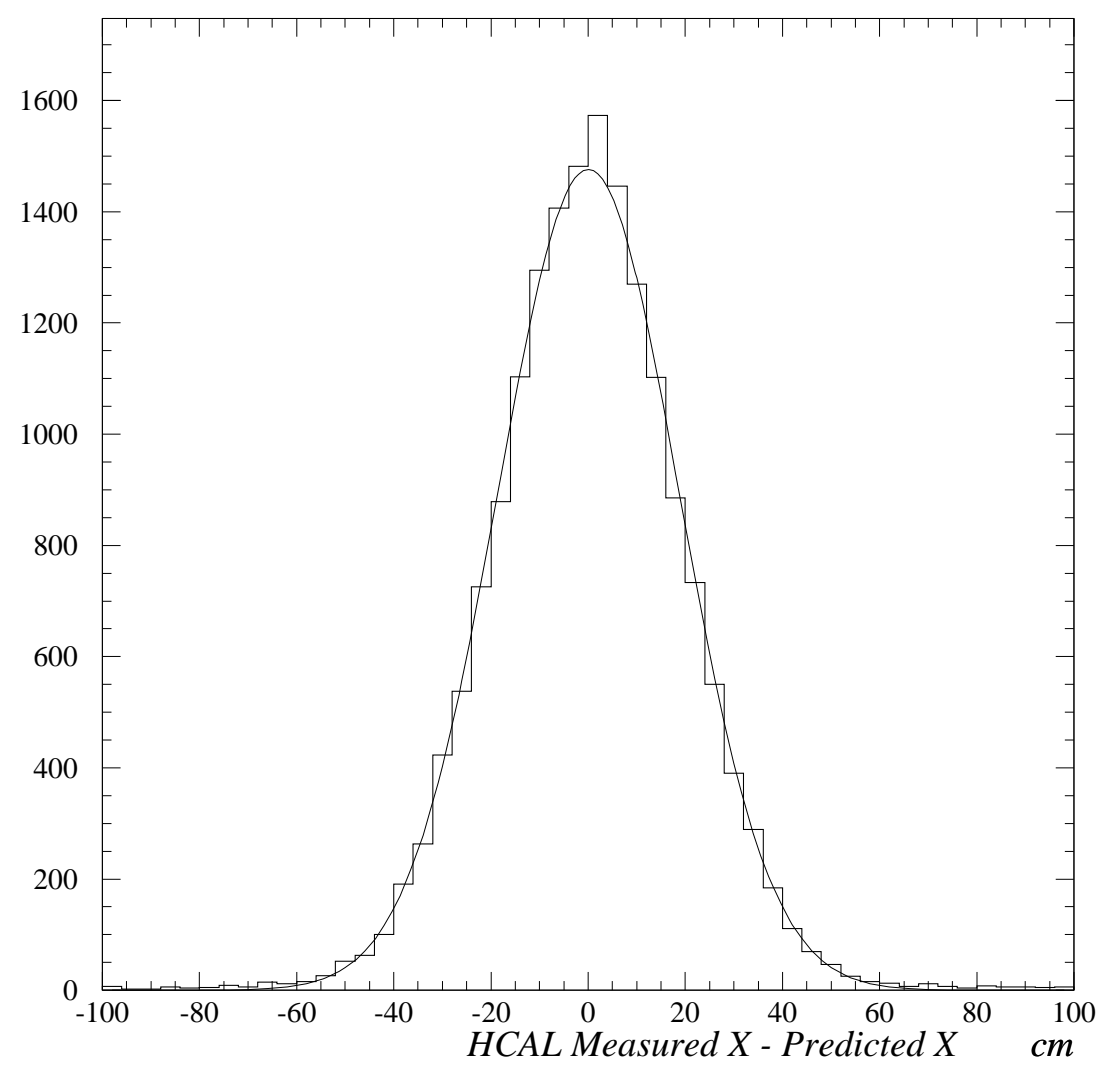

Figure 20: Horizontal position resolution for muons passing through a single hadron calorimeter module 


\subsection{Muon chambers}

The NOMAD muon detector consists of 10 drift chambers previously used in the UA1 experiment [25]. Each chamber has an active area of $3.75 \times 5.55 \mathrm{~m}^{2}$ with two planes of drift tubes in the horizontal and two in the vertical directions. In total there are 1210 drift tubes, each with a maximum drift distance of $7 \mathrm{~cm}$.

The chambers are arranged in pairs (modules) for track segment reconstruction. The first muon station consists of three modules and is placed behind the hadron calorimeter. It is followed by an $80 \mathrm{~cm}$ thick iron absorber and a second muon station of two modules. In 1995 there was a small gap between the muon chambers in station 1. For the 1996 run this gap was closed with several small scintillation counters, the 'muon veto', which are shown in Figs. 1 and 2.

Track segments are reconstructed separately for each station from (typically) three or four hits per projection, see Fig. 21. The measured efficiency for the reconstruction of track segments is $97 \%$.

The chambers are operated with an argon/ethane (40\%/60\%) gas mixture. Their performance is monitored continuously using high-energy muons passing through the detector. The average position resolution for hits is in the range $350 \mu \mathrm{m}$ to $600 \mu \mathrm{m}$ depending on the gas quality. Figure 22 shows the residuals for a typical run.

The average hit efficiency is $92.5 \%$ and the dominant source of the inefficiency $(6.5 \%)$ is due to the dead areas between the drift tubes.

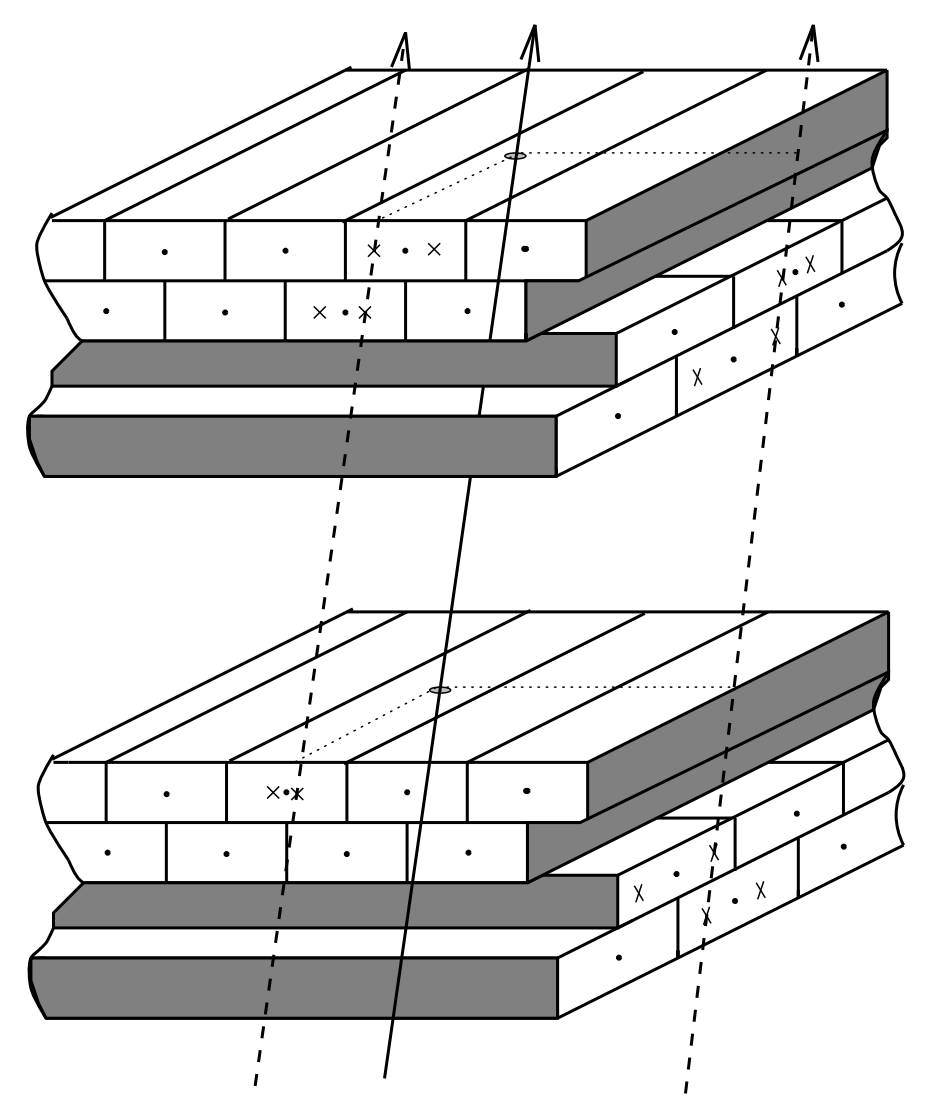

Figure 21: A cut through a muon chamber module illustrating the drift tube arrangement, and the typical hit pattern left by a through-going muon (continuous arrow) and the track segment reconstructed in projection (dashed arrows). 


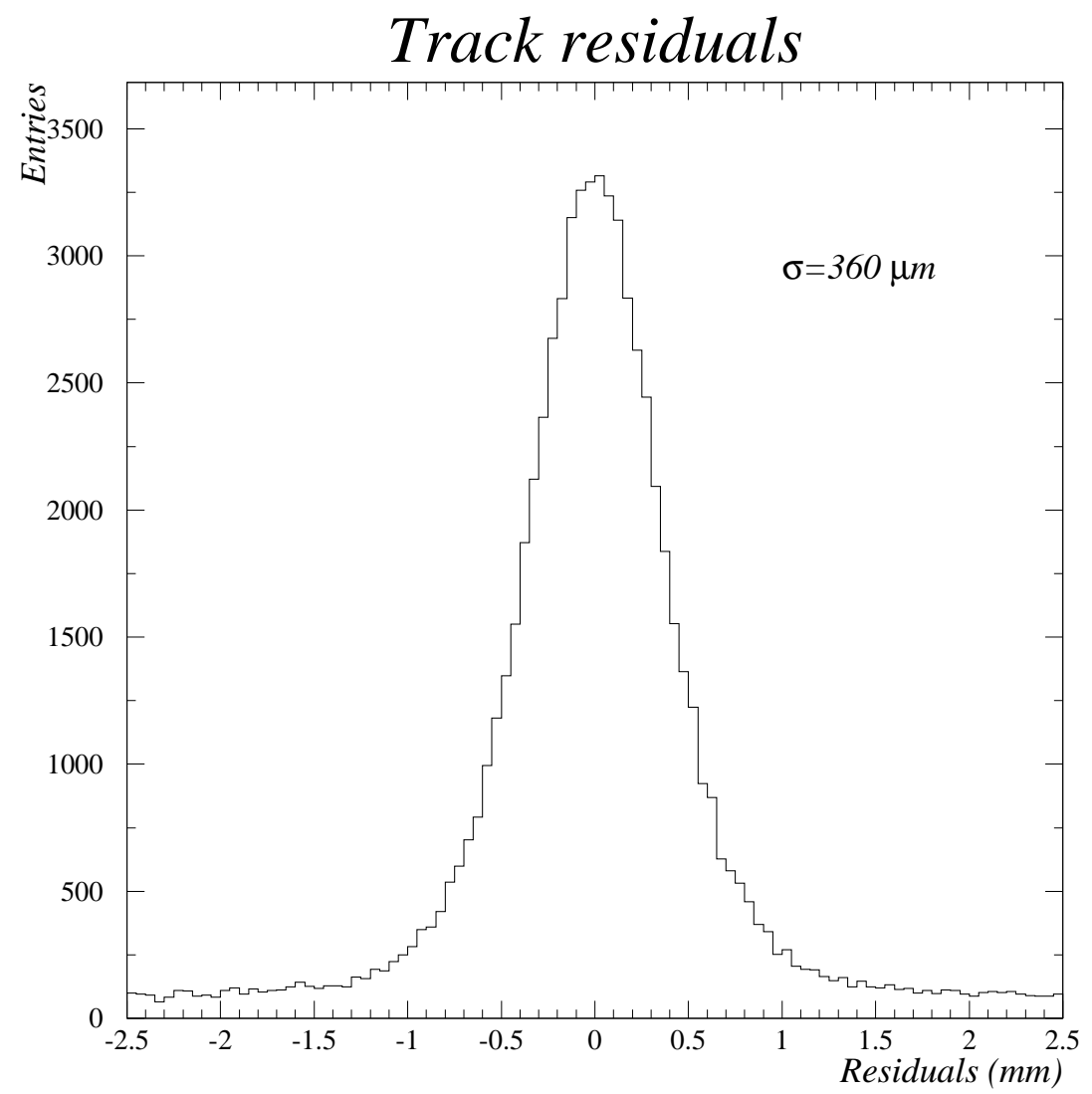

Figure 22: Residuals for 4-point tracks in the muon chambers for a typical run

\subsection{Triggering}

The trigger logic is performed by the VME-based MOTRINO module, which was especially designed for NOMAD. The versatility of the module allows one to operate it in two modes. Six modules function in the trigger-receiver or slave mode and two modules in the trigger-formation or master mode. MOTRINO has the following functionalities [26]:

- Generation of time signals in the master module to synchronize with the SPS neutrino beam cycle. Two gates are generated for the neutrino bursts and one for the flat top.

- Logical combination of up to eight subdetector signals for trigger formation. A maximum of six different trigger types is possible in each of the gates.

- Distribution of the trigger signals to the subdetectors using the module-specific differential bus. The gates and busy signals are also transmitted via this bus.

- Treatment of the busy signals from the different slave modules.

- Storing of the trigger time relative to the SPS cycle in the master and slave modules. The time stamp that each trigger receives serves as a control for data integrity.

- Recording of the status of the trigger bits. The information is stored as soon as the first trigger fires and again, $200 \mathrm{~ns}$ later, to record any additional trigger bit set.

- Determination of the live time for the various trigger types using an external clock which reflects the time structure of the neutrino beam.

- Moderation of trigger rates. This feature, achieved by introducing dead time in a particular trigger, is extensively used during the flat top between neutrino spills in order to adjust individual calibration and alignment trigger rates and thus to limit the total number of events. 
The following triggers were set up for the study of neutrino interactions in NOMAD:

- $\overline{\mathrm{V}} \times \mathrm{T}_{1} \times \mathrm{T}_{2}$. This trigger allows a study of neutrino interactions in the drift chamber target region. At least one hit in both trigger planes $T_{1}$ and $T_{2}$ is required. To prevent triggering on through-going muons, no hit should have occurred in the veto counters $(\mathrm{V})$. The rate for this trigger is $\sim 5.0 / 10^{13}$ p.o.t. and the live time is $(86 \pm 4) \%$. Of these triggers, about 0.5 are potentially interesting candidates for neutrino interactions in the drift chambers. The remaining triggers consist of 'cosmics' (about 1), non-vetoed muons (1.5) and neutrino interactions in the magnet (2.0).

$-\quad \overline{V_{8}} \times$ FCAL. Neutrino interactions in the front calorimeter with an energy deposition of at least 4 m.i.p. fire this trigger. Through-going muons are vetoed by the veto subset $\mathrm{V}_{8}$. On average 6.5 neutrino interactions occur in the FCAL for $10^{13}$ p.o.t. The live time is $(90 \pm 3) \%$.

- $\overline{\mathrm{V}_{8}} \times \mathrm{T}_{1} \times \mathrm{T}_{2} \times$ FCAL'$^{\prime}$. This trigger is set up to study quasi-elastic like events in the FCAL. Such events allow a relative flux measurement of the neutrino beam as a function of the neutrino energy. For this trigger, an energy deposition between 1 and 3 m.i.p. in the FCAL is required. The rate for this trigger is $\sim 1.5 / 10^{13}$ p.o.t. and the live time $(90 \pm 3) \%$.

- $\overline{\mathrm{T}_{1} \times \mathrm{T}_{2}} \times$ ECAL. The electromagnetic calorimeter is also used as a target. Physics topics that will be addressed using events from this trigger include $\nu_{\mu} \rightarrow \nu_{\mathrm{e}}$ and $\nu_{\mu} \rightarrow \nu_{\tau}$ oscillations. An energy deposition of more than $\sim 1.0 \mathrm{GeV}$ in the ECAL enables this trigger, which has an average rate of $2 / 10^{13}$ p.o.t. and a live time of $(88 \pm 3) \%$.

- RANDOM. A random trigger which allows a study of detector occupancy.

Approximately 15 neutrino candidate triggers are taken in each neutrino spill.

In addition various triggers are used in the $2.6 \mathrm{~s}$ long flat-top between the two neutrino bursts. These are used for:

- The drift chamber alignment.

- The calibration of the different subdetectors.

- A measurement of the trigger counter efficiency.

One of the triggers is a selection of electrons, from muon decay or delta rays, which allows a study of the behaviour of electrons in the detector. In total, about 60 triggers are taken in each flat top.

\subsection{Data acquisition system}

NOMAD operates in the burst mode, where triggers arrive in short intervals (spills) separated by relatively long intervals without beam. In order to minimize the dead time, the digitized information is buffered internally on FASTBUS electronics and read out immediately after the end of each spill.

Signals from each subdetector arrive at some combination of three types of FASTBUS modules: twelve-bit charge-integrating ADCs, twelve-bit peak-sensing ADCs, and sixteen-bit TDCs with one nanosecond resolution. Both types of ADCs were designed at CERN, accommodating 64 channels and a 256 event memory. The LeCroy 1876 Model 
100 TDCs provide input for 96 channels with a 64-kilobyte buffer as well as internal zero-suppression. There are a maximum of 11648 channels to read out per event.

Five VME-based boards (FIC 8234 [27]) with Motorola 68040 processors control the readout of the front-end electronics through an extended VME Subsystem Bus (VSB) connected to slave controllers (F68B7) in each of 12 FASTBUS crates. Each FASTBUS controller card provides four megabytes of additional memory for event buffering. The VME controllers perform block transfers of the available data to local buffers, assemble the data into subevents, and check for consistency and integrity. They then pass the subevents through a VME interconnect bus (VIC) to a sixth VME processor (the 'event builder') which assembles all the pieces into complete events together with information about the beam extraction and writes them to one of two nine-gigabyte disks via a highlevel networking package (NIC) developed at CERN. Twice daily, a separate process transfers the data to a tape vault for storage on high capacity DLT tapes. Several Sun Sparc workstations monitor the quality of the data and the status of the detectors.

The data-acquisition software centres around elements known as stages. These stages use the CERN-designed CASCADE [28] software which provides a well-organized framework for DAQ development including scheduling, buffer management, and event access facilities. The arrival of data drives the stages; hence, stages must have at least one input source along with any number of output sources. The standard configuration for NOMAD is shown in Fig. 23.

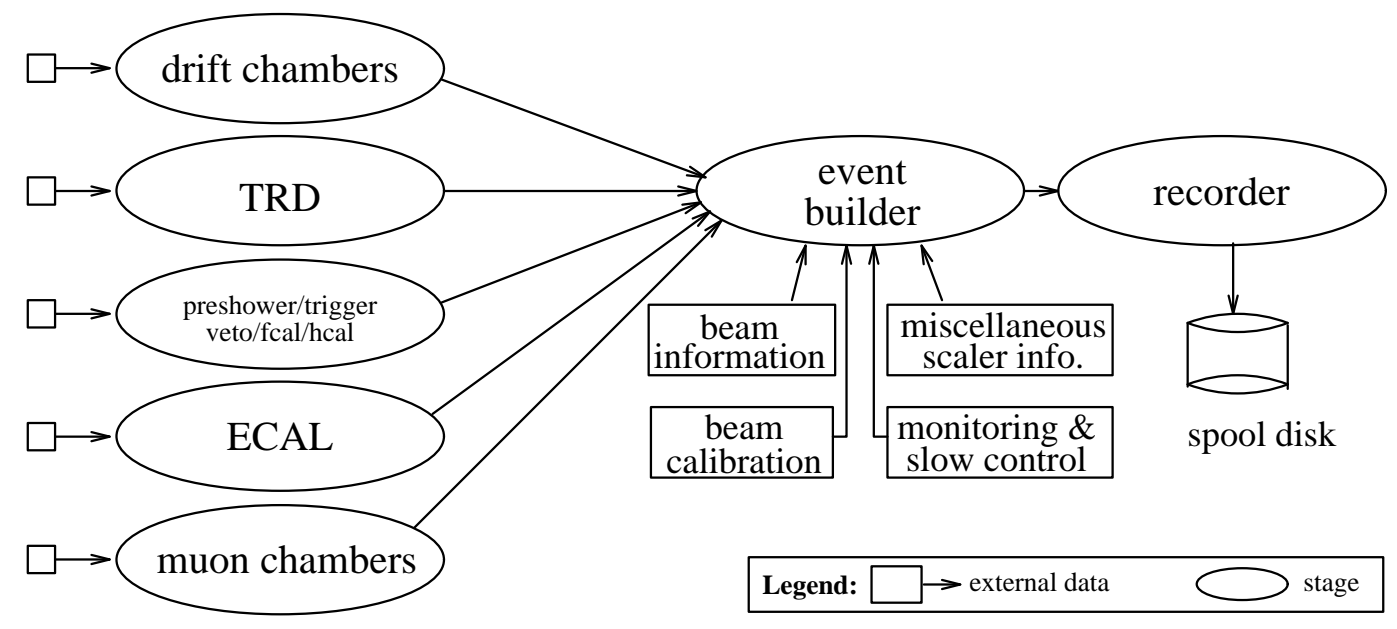

Figure 23: Data-acquisition software configuration

The event-builder stage also asynchronously receives beam calibration data, summaries of monitoring information, and detector status information whenever they change.

The slow control system consists of a Sun workstation and several Apple Macintosh computers running LabView graphical software [29]. The Macintoshes monitor all high and low voltages, gas systems, and temperature probes in the experiment, and pass slow control data to the Sun workstation. Alarms are generated in the event of detector problems. Periodic samples of slow control data, along with all alarm records, are passed from the Sun to the event builder stage and saved for offline use.

Monitoring programs for each of the nine subdetectors as well as for beam, scaler and trigger information connect to the stages via ethernet and generate summary histograms. Shift personnel use the resulting histograms to verify the quality of the data. Additionally, a separate monitoring task reconstructs all the muon events received during the flat top of each SPS cycle and records them in a separate data stream. 
The run-control consists of a finite-state machine implemented in $\mathrm{C}^{++}$with an $\mathrm{X}$ windows interface. It uses a database both to record the current state of all the stages and monitoring programs and to determine rules for transitions between the various states. Periodically, the offline software retrieves summary information for completed runs from this database and combines them with tape information for use during reconstruction and analysis.

The system records over 1.5 megabytes of data per minute, with a typical assembled neutrino event containing around 2000 32-bit words before reconstruction. Additionally, approximately six times this quantity flows through the acquisition in the form of calibration events which are not recorded. In the neutrino spills, the data-acquisition has a typical dead time of $10 \%$ arising from digitizations. The data-taking time lost due to down-time and inter-run transitions is less than $3 \%$.

\section{PERFORMANCE}

Figure 24 shows a candidate $\nu_{\mu} \mathrm{CC}$ event detected in NOMAD. One energetic particle penetrates to the muon chambers, and satisfies all other criteria to be a muon. A hadronic jet of charged particles is clearly visible at the primary vertex, and a photon conversion in the drift chambers is also evident.

Figure 25 shows a candidate $\nu_{\mathrm{e}} \mathrm{CC}$ event detected in NOMAD. One very straight secondary track, identified as an electron by the TRD, is seen to deposit a large amount of energy in a few cells of ECAL, as indicated by the large 'bar' in the event display. Note that in this view a second, positive track appears to be produced at the same space angle as the electron, indicating that the event might be caused by an asymmetric Dalitz decay of a $\pi^{0}$. However the orthogonal view, not shown, indicates that these two tracks are well separated in angle at the primary vertex, which is very unlikely in a Dalitz pair.

\subsection{Identification of $\mathrm{K}_{\mathrm{s}}^{0}$}

$\mathrm{K}_{\mathrm{s}}^{0}$ mesons observed in NOMAD are used as a quality check of the reconstruction program and of the detector performance. Using an algorithm looking for ' $\mathrm{V}^{0}$ ' vertices, $\mathrm{K}_{\mathrm{s}}^{0} \rightarrow \pi^{+} \pi^{-}$and $\Lambda^{0} \rightarrow \mathrm{p} \pi^{-}$decays and $\gamma \rightarrow \mathrm{e}^{+} \mathrm{e}^{-}$conversions are reconstructed.

Figure 26 shows the $\pi^{+} \pi^{-}$mass distribution, where the following selection criteria were applied:

- A pair of oppositely charged tracks, not positively identified as electrons or muons, emerges from a secondary vertex distinct from the primary.

- The reconstructed momentum of the pair was required to point to the primary vertex.

- The invariant mass of the pair, taken to be a $\pi^{+} \pi^{-}$, was required to be greater than $300 \mathrm{MeV} / c^{2}$.

- The invariant mass of the pair, taken to be a $\mathrm{p} \pi^{-}$, was required to differ from the $\Lambda^{0}$ mass by more than $50 \mathrm{MeV} / \mathrm{c}^{2}$.

The $\mathrm{K}^{0}$ peak stands out clearly over a small background. A fit gives a value for the mass of $(497.3 \pm 0.4) \mathrm{MeV} / c^{2}$. The resolution is $11 \mathrm{MeV} / c^{2}$ which is consistent with the measured momentum resolution of NOMAD.

Figure 27 shows the proper distance travelled by the $\mathrm{K}_{\mathrm{s}}^{0}$ before decaying. The fit gives a lifetime of $(892 \pm 65) \times 10^{-13} \mathrm{~s}$. Both the mass and lifetime of the $\mathrm{K}_{\mathrm{s}}^{0}$ given above are consistent with the values given in Ref. [30]. 


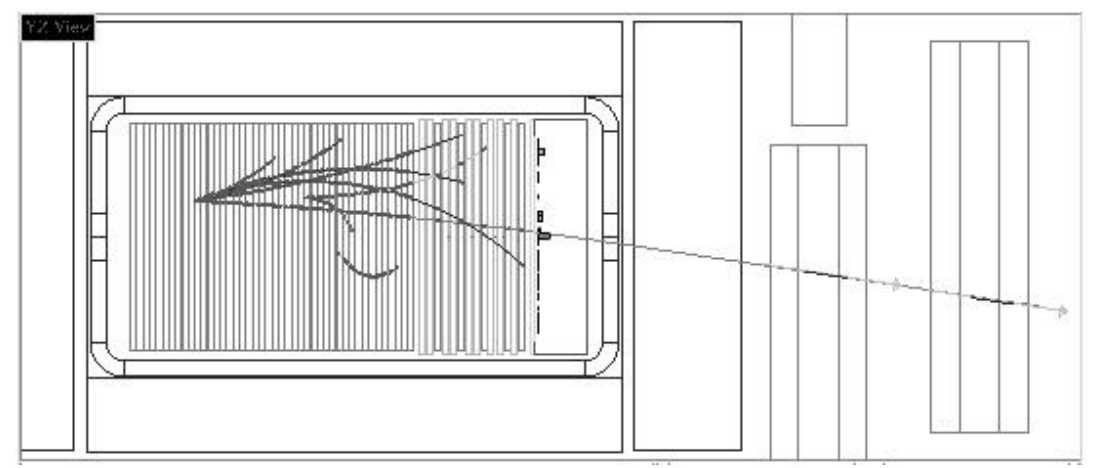

Figure 24: A candidate $\nu_{\mu} \mathrm{CC}$ event; see text for details.

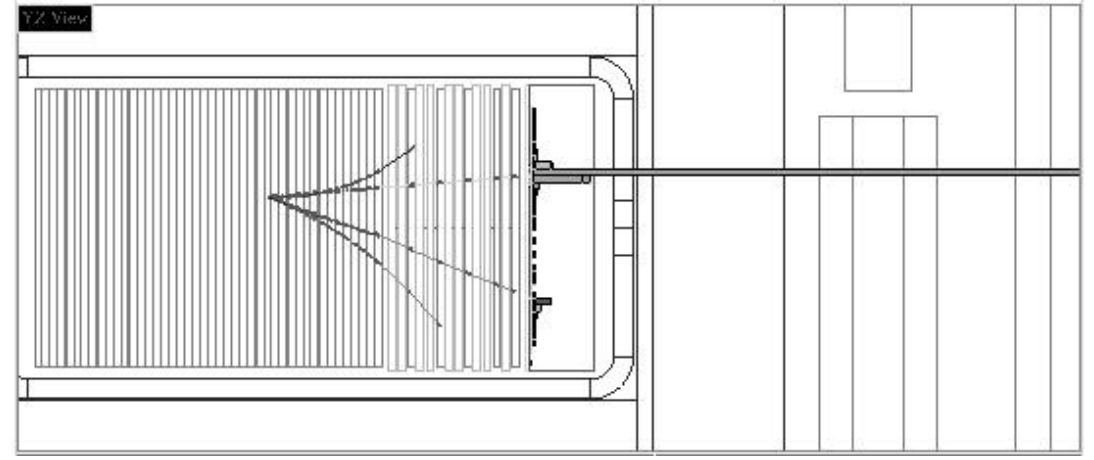

Figure 25: A candidate $\nu_{\mathrm{e}} \mathrm{CC}$ event; see text for details.

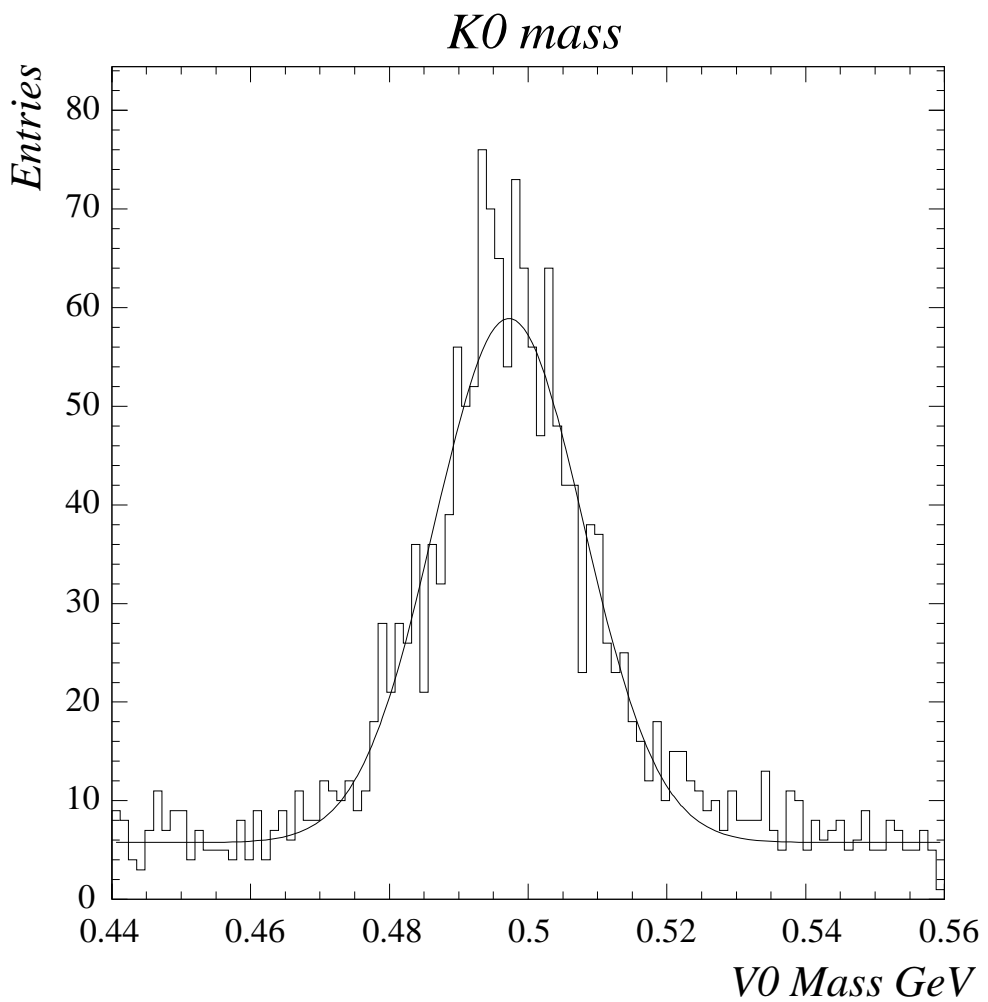

Figure 26: The invariant mass distribution of $\left(\pi^{+} \pi^{-}\right)$pairs from secondary vertices 


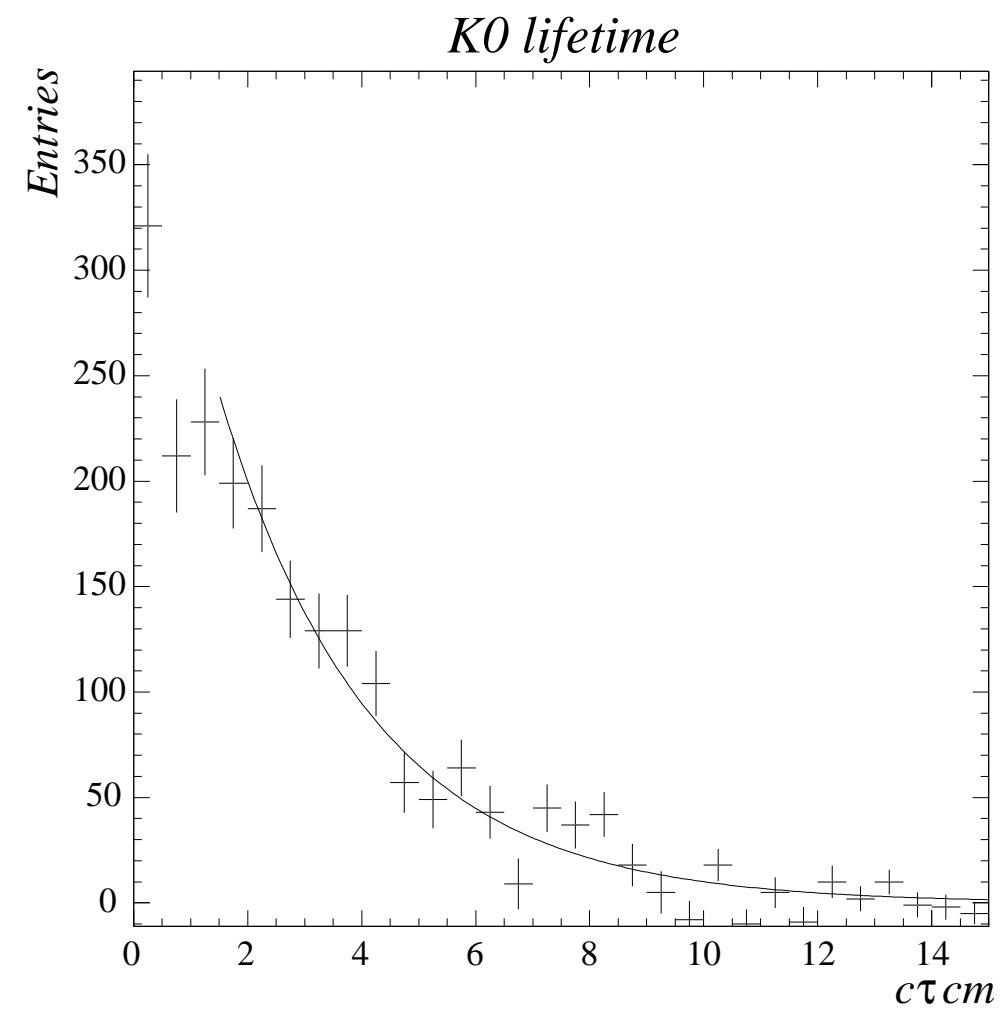

Figure 27: The product $\mathrm{c} \tau$ of $\mathrm{K}_{\mathrm{s}}^{0}$ mesons observed in NOMAD

\subsection{Reconstruction of $\pi^{0}$ mesons}

Gamma-rays from $\pi^{0}$ decays represent the best electromagnetic probe available in NOMAD to test the ECAL response. In spite of the fact that the $\pi^{0}$ flux from neutrino interactions is too small to allow an individual calibration of each ECAL block, the $\pi^{0}$ signal can still be used to test the overall energy response.

Figure 28 shows the $\gamma \gamma$ invariant mass distribution obtained for two-charged-track events with a vertex in the DC region and two neutral clusters in ECAL. The ECAL energy is corrected as explained in Ref. [23]. The peak position is well centred on the $\pi^{0}$ mass and the width $\left(\sigma=11 \mathrm{MeV} / c^{2}\right)$ is in good agreement with the expected resolution.

A similar distribution is obtained requiring one neutral cluster in ECAL and a $\gamma$-ray converted and reconstructed in the DC (Fig. 29). Again the peak position and the width are in agreement with the $\pi^{0}$ mass and the experimental resolution respectively.

\subsection{Muon identification and veto}

The muon chambers in NOMAD are arranged in two 'stations' as described in Section 2.10. Muons are identified if they penetrate more than 8 interaction lengths $\left(\lambda_{\text {int }}\right)$ of absorber material in order to reach muon station 1 , or $13 \lambda_{\text {int }}$ for muon station 2 . For perpendicular incidence, the momentum thresholds to reach the muon chambers (with $50 \%$ probability) are measured to be $2.3 \mathrm{GeV} / c$ for station 1 , and $3.7 \mathrm{GeV} / c$ for station 2 .

The geometrical acceptance in order to hit either of the two stations is about $98 \%$. This number applies to primary muons from charged-current interactions, averaged over their production point in the NOMAD target, and does not include muons which 'range out' in the absorber material. 


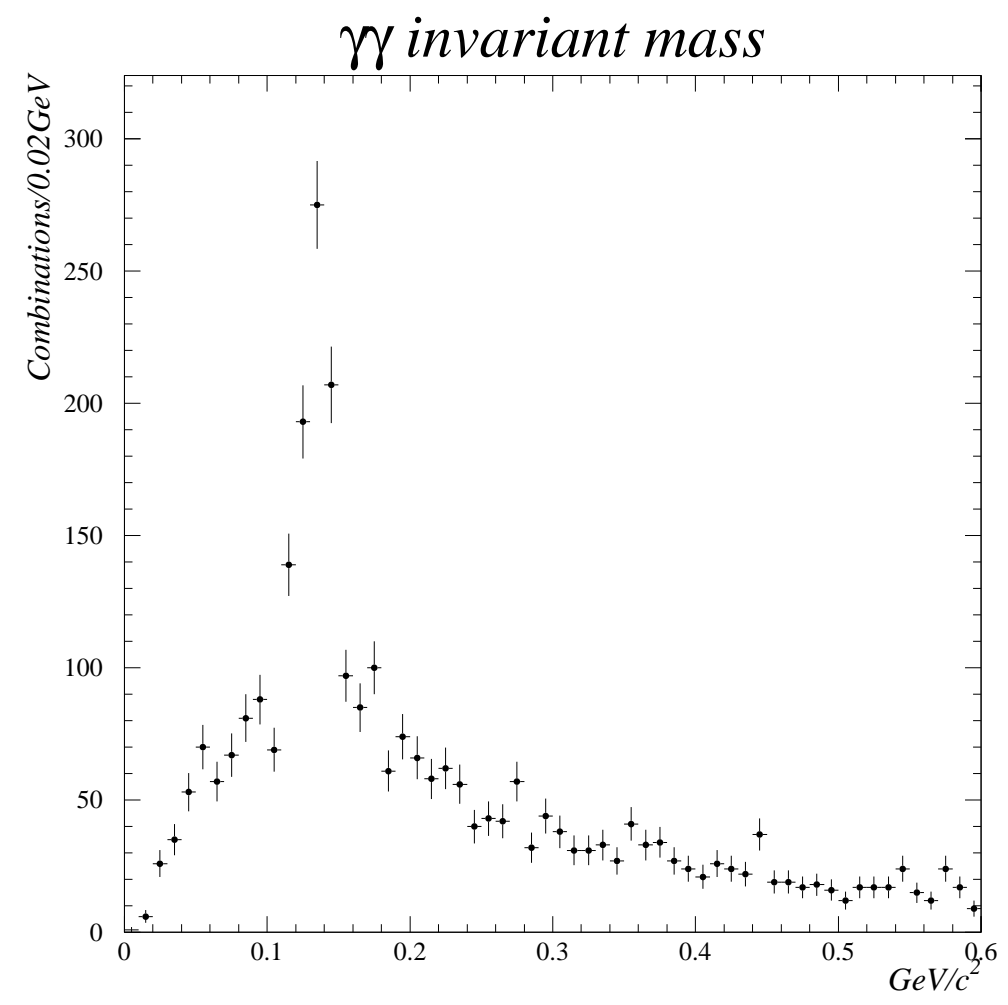

Figure 28: The $\gamma \gamma$ invariant mass where both $\gamma$ energies are measured in ECAL

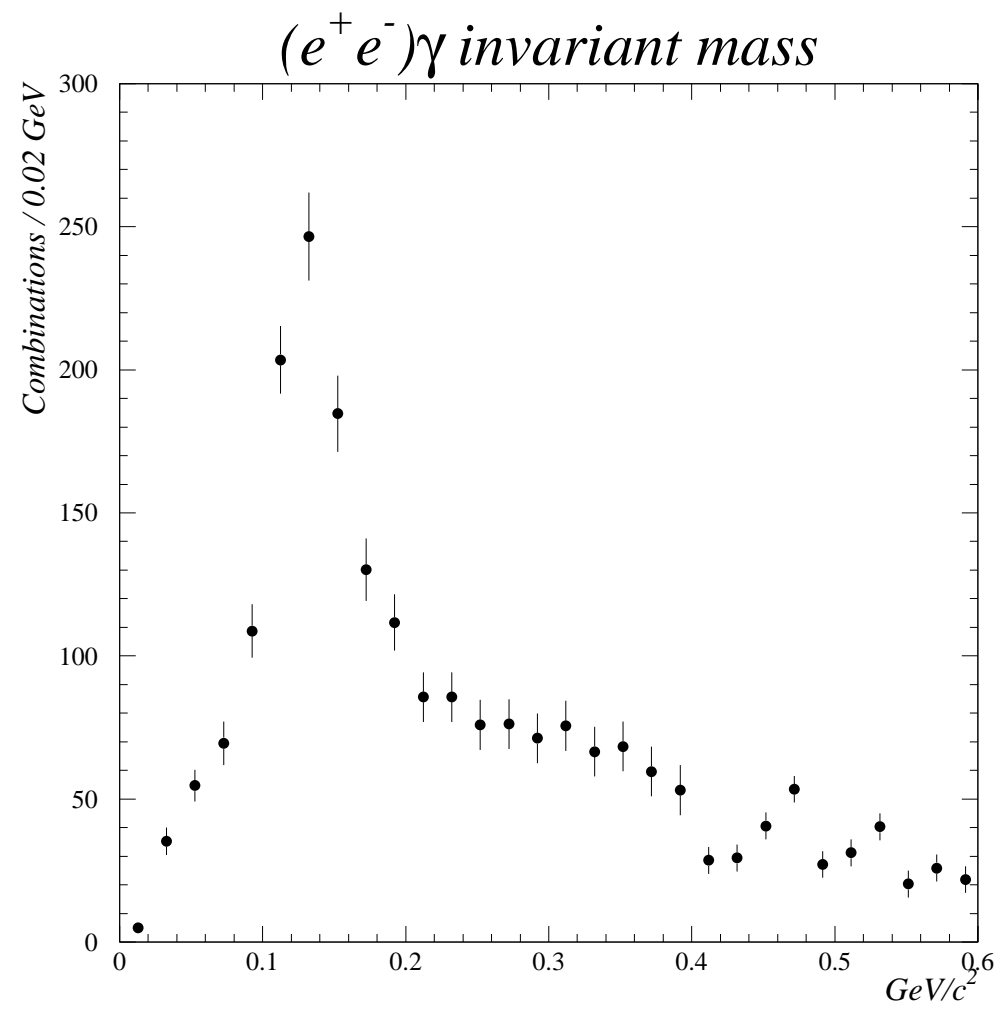

Figure 29: The $\gamma \gamma$ invariant mass where one $\gamma$ converts in the DC and a second one is measured in ECAL 
The muon momentum is measured in the central drift chambers with a precision of typically $3 \%$ for momenta below $20 \mathrm{GeV} / c$, where the error is dominated by multiple scattering. For larger momenta the error slowly rises as measurement errors start to dominate, but the muon charge can be reliably measured for momenta up to $200 \mathrm{GeV} / c$.

A muon typically leaves a signal in the ECAL which is equivalent to a $\approx 550 \mathrm{MeV}$ electromagnetic shower, and a signal in the $\mathrm{HCAL}$ equivalent to $\mathrm{a} \approx 1.5 \mathrm{GeV}$ hadronic shower.

Tracks are reconstructed from the hits in the muon chambers (see Section 2.1) and the extrapolated central drift chamber tracks are matched to these tracks. If this matching satisfies certain quality criteria (e.g. cuts on matching distance and $\chi^{2}$ ), which may vary for different analyses, then the track is identified as having been made by a muon. It is clear from Fig. 30 that the muon reconstruction efficiency is essentially momentumindependent above $5 \mathrm{GeV} / c$.

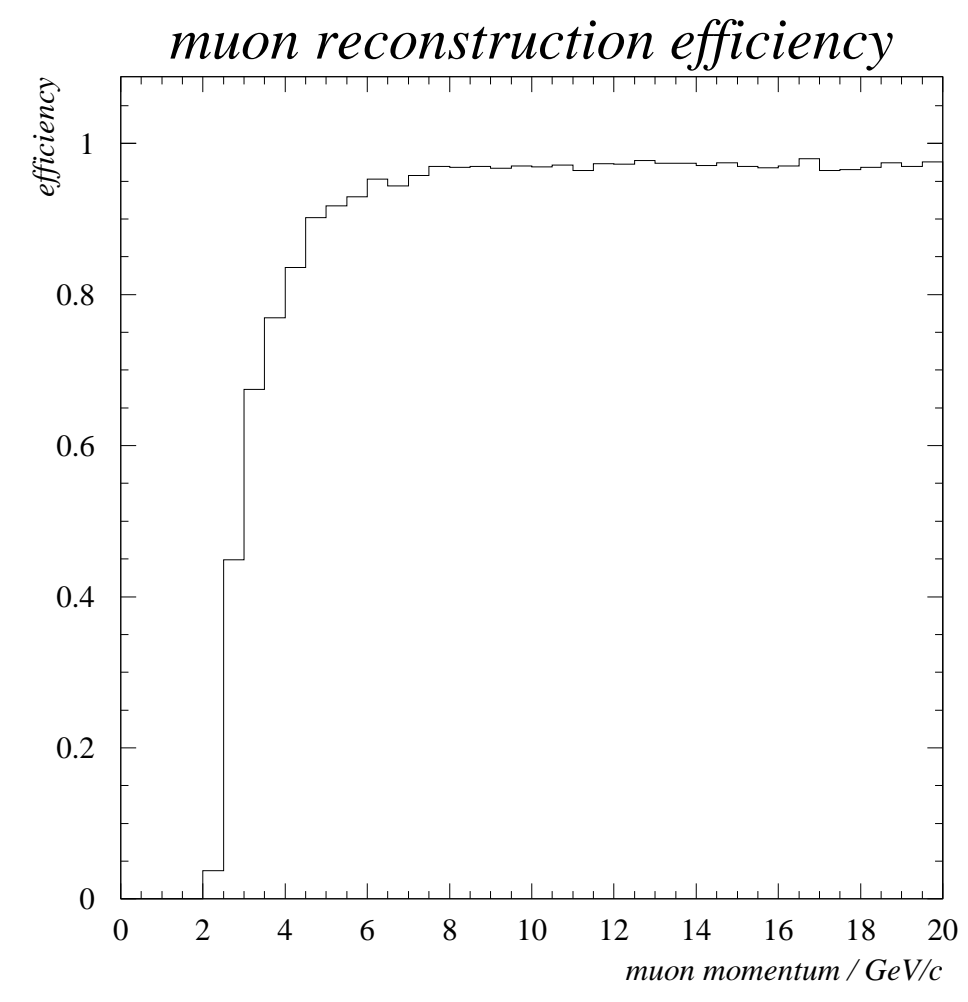

Figure 30: Typical muon reconstruction efficiency as a function of muon momentum for $\nu_{\mu} \mathrm{CC}$ events. This includes tracking, vertex association, the probability for a muon to emerge from the iron absorbers, the geometrical efficiency and the matching efficiency, (multiple scattering).

It is very important for many NOMAD analyses to identify events which do not contain a primary muon. Very low momentum muons can only be eliminated using kinematic criteria, such as $p_{\mathrm{T}}$ cuts. For higher momentum tracks the consistency of the ECAL and HCAL energy deposition can be checked. For muons well above the momentum threshold to reach the muon chambers, the efficiency to detect at least 2 hits is about $98 \%$. As an example, requiring that no muon chamber hits be associated to candidate hadrons within a certain road, and that the energy deposition in the ECAL and HCAL be inconsistent with a minimum-ionizing particle, a residual muon contamination of about $10^{-3}$ can be achieved for hadrons with $p>4 \mathrm{GeV} / c$ with $90 \%$ efficiency. 


\subsection{Electron identification}

Information from the Transition Radiation Detector (TRD), Preshower (PRS), and Electromagnetic Calorimeter (ECAL) is combined to separate electrons from charged $\pi$ mesons.

\subsubsection{Using the transition radiation detector}

The NOMAD TRD (see Section 2.6) is designed to separate electrons from heavier charged particles, mainly pions.

A 'hit' in the TRD is a straw tube with an energy deposition above the pedestal value. A set of hits collected along the road around a drift chamber track is associated to this track. Energy depositions in associated hits are then compared to the expectations for two possible hypotheses: electron (e) and pion $(\pi)$. The momentum of the particle measured in the drift chambers is taken into account to evaluate the expected energy deposition.

Two cases are considered for particle identification:

- A set of hits is produced by a single isolated particle.

- Several particles cross and give signals in the same straw tubes, producing a set of 'shared' hits.

Depending upon the topology of the event, two different identification procedures are applied.

The signature of an isolated particle is defined from a likelihood ratio $\mathcal{L}$ computed from the responses of all of the straw tubes crossed by an incident track:

$$
\mathcal{L}=\sum_{i=1}^{N} \log \frac{P\left(\varepsilon_{i} \mid \mathrm{e}\right)}{P\left(\varepsilon_{i} \mid \pi\right)}
$$

where:

- $\quad N$ is the number of hits assigned to a track.

- $P\left(\varepsilon_{i} \mid\right.$ e) and $P\left(\varepsilon_{i} \mid \pi\right)$ are the probability density functions for an electron e and a pion $\pi$ with a given momentum to deposit the energy $\varepsilon_{i}$ in the $i$-th straw tube.

The distributions of probability density $P\left(\varepsilon_{i} \mid\right.$ e) and $P\left(\varepsilon_{i} \mid \pi\right)$ that a given energy deposition $\varepsilon_{i}$ belongs to an electron or pion have been obtained from detailed simulation, extensive test beam, and in situ measurements (for details see Ref. [21]). The desired electron efficiency defines a threshold on the total likelihood ratio, see Fig. 31. An electron (pion) is associated with a likelihood ratio above (below) this threshold. A pion rejection better than $10^{3}$ at $90 \%$ electron efficiency has been achieved for isolated particles crossing all nine TRD modules in a wide momentum range from $1 \mathrm{GeV} / c$ to $50 \mathrm{GeV} / c$.

About $25 \%$ of $\nu_{\mathrm{e}} \mathrm{CC}$ interactions in NOMAD lead to tracks having 'shared' hits in the TRD. An assignment of a total deposited energy in shared hits to each of the non-isolated tracks would lead to particle misidentifications. For example, more than 95\% of two pions with all nine hits shared would fake an electron signal.

The identification procedure for non-isolated particles takes into account the number of tracks producing each hit and their momenta. While for an isolated track a decision is made between two hypotheses $\mathrm{e}(p)$ and $\pi(p)$, for non-isolated tracks one has to consider four hypotheses $\pi\left(p_{1}\right) \cdot \pi\left(p_{2}\right), \mathrm{e}\left(p_{1}\right) \cdot \pi\left(p_{2}\right), \pi\left(p_{1}\right) \cdot \mathrm{e}\left(p_{2}\right)$ and $\mathrm{e}\left(p_{1}\right) \cdot \mathrm{e}\left(p_{2}\right), p_{1}$ and $p_{2}$ being the momenta of the tracks. A detailed description of the procedure can be found in Ref. [21], and a summary of the identification algorithm is presented below. 
- If two tracks have at most three 'shared' hits, the particle identification procedure for isolated tracks is applied to 'non-shared' hits only.

- If two tracks have more than three 'shared' hits, four likelihood estimators are computed for the energy depositions in 'shared' hits from the corresponding two-particle probability distributions. The decision on the nature of each of the two particles is made by selecting the hypothesis corresponding to the maximum likelihood value.

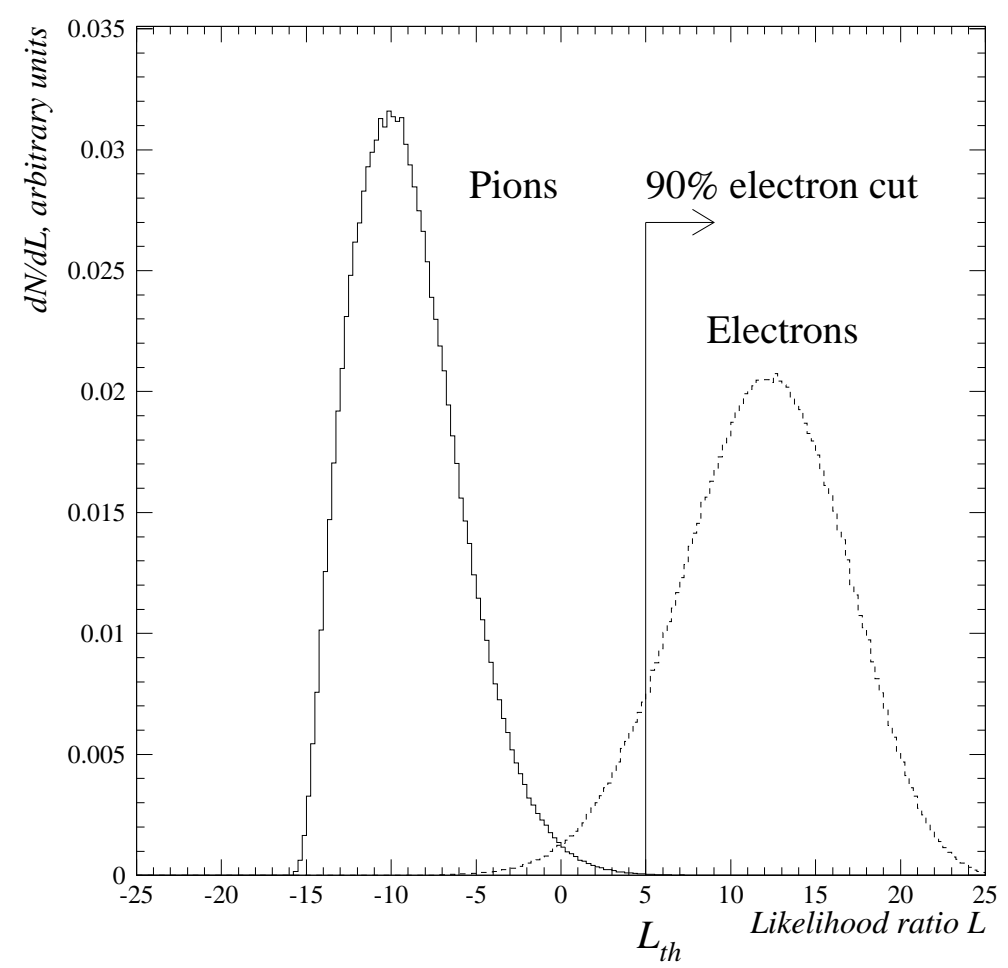

Figure 31: The likelihood ratio distributions for pions and electrons with track momenta $10 \mathrm{GeV} / c$ crossing nine TRD modules (Monte Carlo simulation). Pion rejection is better than 1000:1 at $90 \%$ electron efficiency.

The main goal of the algorithm developed for the identification of non-isolated particles is to reduce the number of fake electrons caused by non-isolated hadrons. It allows a correct identification of $84 \%$ of the non-isolated pions produced in $\nu_{\mathrm{e}} \mathrm{CC}$ interactions in NOMAD, with $15 \%$ of $\pi \cdot \pi$ combinations being misidentified as e $\cdot \pi$ and only $1 \%$ as $\mathrm{e} \cdot \mathrm{e}$.

The performance of the identification algorithms has been studied on a sample of muons producing energetic $\delta$-ray electrons $\left(p_{\delta}>500 \mathrm{MeV} / c\right)$ selected from NOMAD data (Section 2.6). Using the electron identification algorithm for isolated tracks, $(86 \pm 3) \%$ of $\delta$-ray electrons were correctly identified at a $10^{3}$ level of muon rejection, which agrees with the expectations for the electrons in the range from 0.5 to $2.5 \mathrm{GeV} / c$. The identification procedure for non-isolated tracks has been tested by summing the energy depositions of muons and their associated $\delta$-ray electrons. About $(72 \pm 3) \%$ of the e $\cdot \mu$ combinations were properly identified, which is in agreement with the $75 \%$ expected.

The NOMAD TRD reaches a $10^{3}$ pion rejection factor for isolated tracks in the $1 \mathrm{GeV} / c$ to $50 \mathrm{GeV} / c$ momentum range with a $90 \%$ electron detection efficiency. The algorithm developed for the identification of non-isolated tracks allows the number of misidentified particles to be reduced, particularly in large-multiplicity events. 


\subsubsection{Using the preshower and the electromagnetic calorimeter}

A PRS prototype consisting of two layers of 10 tubes each was exposed to beams of electrons and $\pi$ mesons at the CERN PS and SPS accelerators. Based on the data obtained, a procedure was developed for electron identification. The PRS pulse-height (measured in m.i.p.) was required to be larger than:

$$
0.836+6.86 \ln (E)-0.22(\ln (E))^{2}
$$

where $E$ is the energy of the particle in $\mathrm{GeV}$, corrected for linearity and for the energy loss in the PS, as explained in Ref. [23].

For energies greater than $4 \mathrm{GeV}$ this yields an efficiency of $90 \%$ with a residual $\pi$ contamination smaller than $10 \%$.

The $\pi$ /e separation is substantially improved when ECAL is used in association with the PRS. Using a test-beam set-up comprising PRS and ECAL prototypes, the response to both electrons and pions was measured. Figure 32 shows the scatter plots of PRS vs. ECAL pulse-height for $5 \mathrm{GeV}$ electrons and pions. The rectangular regions in the figure correspond to events in which the energy deposited by electrons is consistent with the beam energy within the resolution of ECAL, and the PRS pulse-height satisfies the condition described above. A rejection factor against pions of about $10^{3}$ is obtained in the energy range 2-10 GeV, while retaining an overall efficiency of $90 \%$ to detect electrons. An additional rejection factor of about 2-3 has been obtained using the information on energy sharing among nearby towers in test-beam data [23].
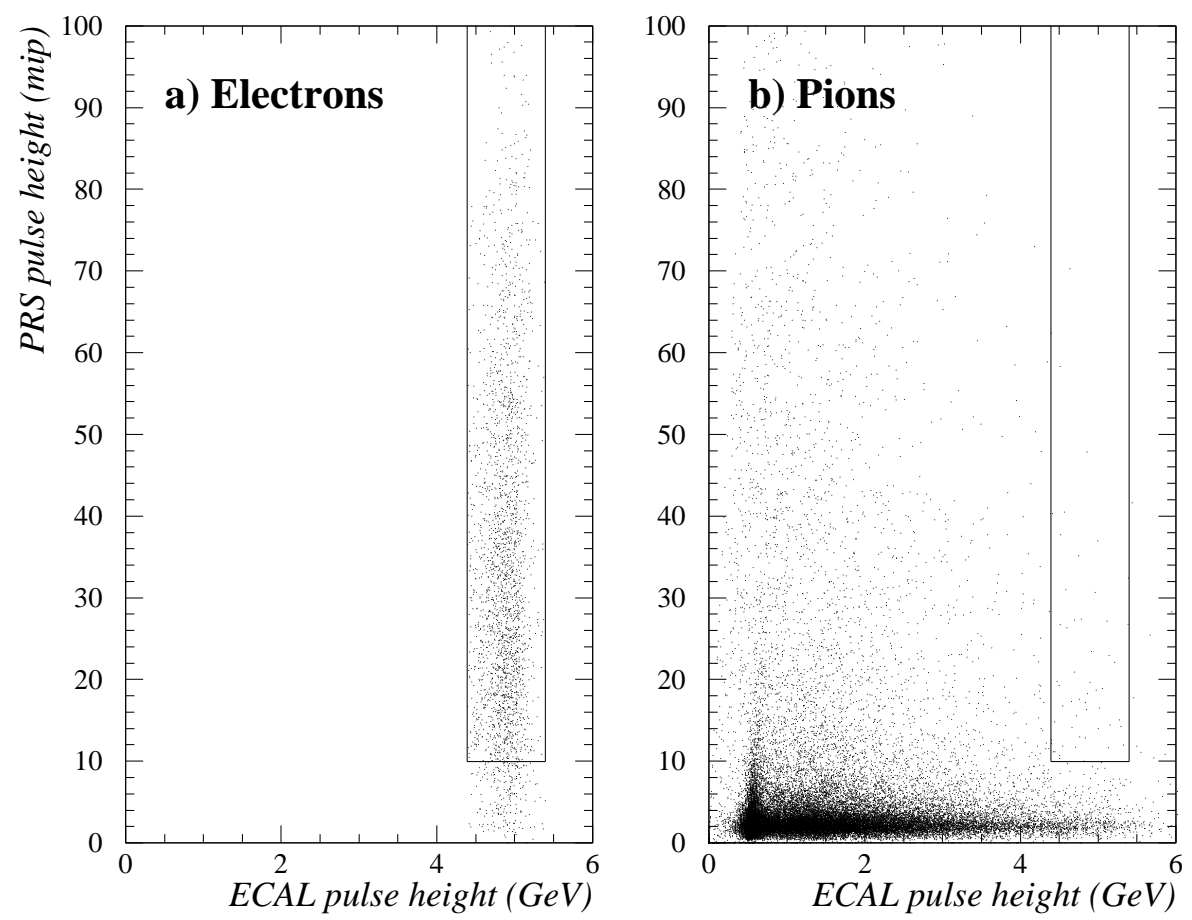

Figure 32: Scatter plots of the PRS vs. ECAL signals for $5 \mathrm{GeV} / c$ incident electrons (a) and pions (b)

\section{CONCLUSIONS}

The NOMAD detector described in this paper collected data in 1995 and 1996 and has accumulated about $600000 \nu_{\mu} \mathrm{CC}$ events resulting from the interactions of $2.25 \times 10^{19}$ protons incident on the beryllium target. The data collection is continuing in 1997 and 
the possibility of extending the run in 1998 has been raised. All the detector components have been shown to function well. The reconstruction of individual particles produced in neutrino interactions and the identification of muons and electrons has been proved to be feasible. The search for $\nu_{\tau}$ appearance is therefore proceeding as planned. Preliminary results based on a reduced data sample have already been presented at conferences. In addition, the data will permit the study, with enhanced statistics, of more conventional processes in neutrino interactions previously made in bubble chambers.

\section{Acknowledgements}

We would like to thank A. Beer, C. Détraz, G. Fetchenhauer, G. Gallay, E. Lessmann, J. Mulon and S. Soulié for their invaluable technical help. We are grateful to P. Farthouat for providing advice on the design of the electronics. We acknowledge the contributions from G. Linser, responsible for the experimental hall, and from H.D. Graskamp for the operation of the magnet. We thank J.M. Maugain and his team for the smooth operation of the West Area Neutrino Facility. We are grateful to the SPS operation crew for the superb performance of the accelerator and of the proton extraction system.

The Australian groups would like to thank the Australian Research Council (ARC) and the Department of Industry, Science and Technology (DIST) for their support.

The French groups thank the Institut National de Physique Atomique et des Particules (IN2P3) and the Commissariat à l'Energie Nucléaire for their financial contributions, and the technical staff of the three participating laboratories for their very competent work.

The Italian groups would like to thank the Istituto Nazionale di Fisica Nucleare (INFN) for financial support; the technical staff of the Cosenza, Firenze, Padova, Pavia and Pisa are gratefully acknowledged for their contributions.

The Johns Hopkins group was supported by the U.S. National Science Foundation under grant PHY-9526278. The work of the Harvard and U.C.L.A. groups was supported in part by the U.S. Department of Energy.

The Dortmund group would like to thank the BMBF for support under contract 056 DO52 I. Thanks are also due to H. Noffke, N. Koch, T. Villet and the staff of their workshops for their excellent work.

The Lausanne group acknowledges the financial support of the Fonds National Suisse de la Recherche Scientifique.

The Dubna group acknowledges the financial support of the Russian Foundation for Fundamental Research under grant 96-02-18562.

The Moscow group gratefully acknowledges the support of the Institute for Nuclear Research of the Russian Academy of Sciences.

The LAPP, PARIS VI and VII and JINR groups thank the French Ministère de l'Education Nationale, de l'Enseignement supérieur et de la Recherche for its support through the creation of the Réseau de Formation et de Recherche JINR-Dubna, LAPPAnnecy, and LPNHE-Paris VI and VII.

Many of the the graduate students in NOMAD were supported by grants from their respective national agencies, which are gratefully thanked. A. Bueno thanks D.G.I.C.Y.T. and M.E.C. for financial support. 


\section{References}

[1] P. Astier et al., Search for the oscillation $\nu_{\mu} \rightarrow \nu_{\tau}$, CERN-SPSLC/91-21, CERNSPSLC/91-48, CERN-SPSLC/91-3.

[2] The magnet was previously used in the UA1 experiment. See: M. Barranco-Luque et al., Nucl. Instr. and Meth. A176 (1980) 175.

[3] G. Acquistapace et al., CERN-ECP/95-14 (July 1995).

[4] L. Casagrande et al., The alignment of the CERN West Area Neutrino Facility, CERN 96-06 (August 1996).

[5] H. Butler et al., CERN-ECP/93-22 (November 1993).

[6] M. Giesch, S. Van der Meer, G. Pluym and K.M. Vahlbruch, CERN-NPA-Int 63-26.

[7] E. Heijne, Muon flux measurement with silicon detectors in the CERN neutrino beams, CERN 83-06 (July 1983).

[8] GEANT: Detector description and simulation tool, CERN Programming Library Long Writeup W5013.

[9] SPY Collaboration, G. Ambrosini et al., CERN-SPSLC/96-01.

[10] B. Van de Vyver and P. Zucchelli, CERN-PPE/96-113 (August 1996).

[11] M.C. Gonzalez-Garcia and J.J. Gomez-Cadenas, CERN-TH/96-220 (August 1996).

[12] The scintillation counters and electronics used in the veto were previously used in the ' $n \bar{n}$ 2' experiment at I.L.L. Grenoble: M. Baldo-Ceolin et al., Z. Phys. C63 (1994) 409.

[13] A Cavestro et al., Nucl. Instr. and Meth. A 305 (1991) 488-491.

[14] The scintillators and photomultipliers were previously used in the CDHS experiment: M. Holder et al., Nucl. Instr. and Meth. 148 (1978) 235. Test measurements showed that the quality of the material was still satisfactory.

[15] W.K. Sakumoto et al., Nucl. Instr. and Meth. A294 (1990) 179.

[16] M. Anfreville et al.,The drift chambers of the NOMAD detector, to be submitted to Nucl. Instr. and Meth. (1997).

[17] Hamamatsu R2490-05; Hamamatsu product catalogue.

[18] T. Fazio, Le détecteur à rayonnement de transition de l'expérience NOMAD pour l'identification du $\tau$ dans le canal $\tau^{-} \rightarrow \mathrm{e}^{-} \overline{\nu_{\mathrm{e}}} \nu_{\tau}$, thèse pour obtenir le titre de Docteur de l'Université Joseph Fourier - Grenoble 1 (April 1995).

[19] G. Bassompierre et al., A large area transition radiation detector for the NOMAD experiment, to be submitted to Nucl. Instr. and Meth. (1997).

[20] G.M. Garibian, JETP 6 (1958) 1079;

G.M. Garibian, JETP 10 (1960) 372;

K.A. Barsukov, JETP 10 (1960) 787;

G.M. Garibian et al., Nucl. Instr. and Meth. 125 (1975) 133.

[21] G. Bassompierre et al., Performances of the NOMAD transition radiation detector, to be submitted to Nucl. Instr. and Meth. (1997).

[22] D. Autiero et al., Nucl. Instr. and Meth. A372 (1996) 556.

[23] D. Autiero et al., Nucl. Instr. and Meth. A387 (1997) 352.

[24] D. Autiero et al., Nucl. Instr. and Meth. A373 (1996) 358.

[25] K. Eggert et al., Nucl. Instr. and Meth. A176 (1980) 217. 
[26] J. Altegoer et al., The trigger system of the NOMAD experiment, to be published in Nucl. Instr. and Meth.

[27] Creative Electronic Systems, Geneva, CH.

[28] Y. Perrin et al., CASCADE: a toolkit for the construction of distributed, real-time, data-acquisition systems, CERN-ECP/93-13 (October 1993).

[29] LabView User Manual for Sun (September 1994 Edition), National Instruments Corporation, Austin, TX, 1992, 1994.

[30] Particle Data Group, Phys. Rev. D54 (1996) 412. 